\title{
Quantum Groups from Path Integrals
}

\author{
DANIEL S. FREED \\ Department of Mathematics \\ University of Texas at Austin
}

January 25, 1995

The goal of these lectures is to explain how quantum groups arise in 3 dimensional topological quantum field theories (TQFTs). Of course, "explain how" is not the job of science, and perhaps you will find other explanations more satisfying. Let me explain!

What is a 3 dimensional TQFT? At the very least it gives a topological invariant of 3 dimensional manifolds. That is, to each 3 -manifold $X$ it assigns a complex number $Z_{X}$ and if the invariants for $X$ and $X^{\prime}$ are different $\left(Z_{X} \neq Z_{X^{\prime}}\right)$, then $X$ and $X^{\prime}$ are not diffeomorphic. A 3 dimensional TQFT also gives invariants of knots and links. For example, if $K$ is a knot in ordinary 3 -space, then we get a set of numerical invariants $\left\{I_{K}(\alpha)\right\}$ indexed by some finite set. The Jones polynomial of a knot, and similar polynomial invariants of knots, fit into this picture. As a historical note, Vaughan Jones [J] introduced his polynomial invariant in the mid 80s before the advent to topological quantum field theories. Those were introduced by Edward Witten in 1987 (following a suggestion of Michael Atiyah), first in 4 dimensions to give a quantum field theoretic interpretation of Donaldson's invariants of 4manifolds. A few years later [W] he introduced a 3 dimensional TQFT which reproduces the Jones polynomial and which is our concern here. The classical action of this field theory is the Chern-Simons invariant, which was introduced into geometry in the early 1970s. As a mathematician I must immediately point out that Witten's methods, involving the path integral, are far from an established part of rigorous 1990s mathematics.

Shortly after Witten's paper on quantum Chern-Simons invariants, Reshetikhin and Turaev [RT1] showed how to start with extremely complicated algebraic datacalled a quantum group - and again produce the Jones polynomial and its generalizations. (Their work is completely rigorous.) Subsequently, they showed [RT2] how to use the same data to construct invariants of 3-manifolds. The construction of a complete TQFT from this algebraic data, which involves more than invariants of 3-manifolds and knots, has been folklore ever since and by now is completely written down $[\mathrm{T}]$. I remark here that instead of starting with a quantum group, one can start with certain "categorical" data instead.

1991 Mathematics Subject Classification. 81R50, 81-01, 58Z05.

The author is supported by NSF grant DMS-9307446, a Presidential Young Investigators award DMS-9057144, and by the O'Donnell Foundation. 
Now I can explain my point of view in these lectures. The algebraic data of either a quantum group or its categorical equivalent is extremely complicated! One could hardly guess in advance that such data can produce invariants of knots and 3-manifolds. Nor can one easily construct algebraic data satisfying the necessary hypotheses. By contrast the classical Chern-Simons action is beautiful and simple! It is relatively easy to write down. One sees from the beginning that Lie groups enter the picture in a fundamental way. And if you are willing to accept the path integral (you shouldn't!), then you have a nice geometric construction of the Jones polynomial and related 3-manifold invariants. This leads us to pose the following.

Problem: Start with the Chern-Simons action and construct the quantum group which gives the same 3-manifold and knot invariants.

My goal is to explain how to do this in a simple case. As I said, the Chern-Simons theory starts with a compact Lie group $G$ (and a piece of topological data which I'll explain later). The Jones polynomial concerns the case $G=S U(n)$ for variable $n$. In the simple case we treat $G$ is a finite group. This was first considered by Dijkgraaf and Witten [DW]. The major simplification here is that the path integral is a finite sum, rather than an integral over an infinite dimensional space, so is rigorously defined. So we immediately get a 3-manifold invariant, though it is rather simple and relatively uninteresting. The knot invariants are possibly more interesting; I don't believe that they have been investigated fully. In any case our interest is in the quantum group and our strategy is this: We exploit the fact that the path integral is well-defined to introduce generalizations of the path integral. Thus one ingredient in a 3 dimensional TQFT is a "quantum Hilbert space" $E(Y)$ for every surface $Y$. In usual quantum field theories it is constructed by canonical quantization. In our simple model we show how to get it by an exotic path integral. Something is immediately very strange - the result of an integration is a Hilbert space! Even more strange is the path integral we introduce for a 1-manifold, i.e., for a circle $S$. There is where we will see the quantum group emerging. In fact, the quantum groups we compute this way were written down in a paper of Dijkgraaf, Pasquier, and Roche [DPR]. They did not related it to the Chern-Simons invariant. It was a conjecture of Altschuler and Coste $[\mathrm{AC}]$ that these quantum groups construct (via the Reshetikhin-Turaev prescription) the invariants of the finite group ChernSimons theory. Our methods prove this conjecture.

This, then, is our strategy. In a $d$ dimensional field theory, where usually the classical action is only defined for fields in $d$ dimensions, we will generalize the classical action to fields on manifolds of dimension less than $d$. We then introduce a corresponding generalization of the path integral for these exotic classical actions. Of course, one is immediately led to ask whether our constructions can be generalized, at least heuristically, in Chern-Simons theory with continuous gauge group, or possibly in other quantum field theories. At this writing I do not know the answer to this question.

The mathematics here is complicated and abstract, but not difficult. I have already tried my hand at several accounts. The original paper, with all of the computations, is [F1]. Previous joint work with Frank Quinn [FQ] discusses the basic theory in more detail. The brief [F2] gives a heuristic account of our extension of TQFT (without mentioning path integrals or the classical theory) as well as a discussion of central extensions (which arise in Chern-Simons theory with continuous gauge groups) and invariants of "framed tangles." The conference proceedings [F3] 
contains a heuristic explanation of our generalized path integrals as well as a brief idea of how some of this structure appears in characteristic numbers in topology. The summer school notes [F4] I wrote a few years ago give a more leisurely introduction to the basics of finite group Chern-Simons theory. For these summer school notes I will unabashedly cut and paste text and pictures from my previous writings! Much of the work I will leave for you in the form of guided exercises. Many of the exercises are not directly related to the topic of these lectures, but perhaps you will find them useful anyhow.

\section{§1: Classical Field Theory}

We begin with a discussion of the basic ingredients in a classical field theoryspacetimes, fields, action. This is mostly to fix the ideas and notation since these same ingredients in the finite group Chern-Simons theory may otherwise appear exotic. We then discuss the Wess-Zumino-Witten action in 2 dimensions in some detail. Here we introduce an extension of the idea of the classical action of a field theory. Although this particular example is not the subject of our lectures, it is a familiar example and hopefully the geometry we discuss is easily accessible.

\section{Classical Actions.}

A field theory has a particular dimension attached to it, which we call ' $d$ '. The standard examples of field theories have $d=4$ and take place on Minkowski space. We allow more general examples. This means first of all that $d$ is not necessarily $4 .^{1}$ Also, we allow spacetimes which are curved manifolds. Generally we take them to be compact, with or without boundary. Notice the terminology we will often use: A manifold is called closed if it is compact and has no boundary. Now Minkowski space carries a metric whose isometry group is the Poincaré group, and one usually requires that the the theory have the Poincaré group as a symmetry. We will generalize this considerably. Namely, a given field theory takes place on manifolds with some specified extra structure. This may be topological (e.g. an orientation or spin structure) or may be geometric (a conformal structure or a metric). Thus usual relativistic field theory should be thought of as taking place on Lorentz manifolds. We allow field theories on Riemannian manifolds and also on manifolds with less rigid structure. A field theory based on Riemannian manifolds is called a Euclidean field theory, one based on manifolds with a conformal structure is a conformal field theory, and one based on manifolds with only some topological structure is a topological quantum field theory. We can be even more adventurous, of course. We might allow singular manifolds, for example. For $d=1$ there are interesting field theories defined on graphs. Or we might allow supermanifolds. Or we might take more abstract sorts of spaces.

So the first ingredients of a classical field theory are a dimension and a class of spacetimes, ${ }^{2}$ i.e., manifolds of that dimension. The next ingredient is a space of fields $\mathcal{C}_{X}$ for each spacetime. This is usually a set of local functions on $X$ if we interpret 'function' liberally enough. The main object of study is the classical action (or simply, action) which is usually a real-valued function function on the space of fields:

$$
S_{X}: \mathcal{C}_{X} \longrightarrow \mathbb{R}
$$

\footnotetext{
${ }^{1}$ In the Chern-Simons example of most interest to us $d=3$.

${ }^{2}$ We use the word 'spacetimes' even though in our context the words 'space' and 'time' may not have much significance.
} 
Typically, if a field is denoted $\phi$, there is a lagrangian density $L_{X}(\phi)$ whose value at $x \in X$ only depends a finite number of derivatives of the field at $x$ and on a finite number of derivatives of the geometric data (such as a metric) on $X$ at $x$. Then the action is the integral of the Lagrangian density:

$$
S_{X}(\phi)=\int_{X} L_{X}(\phi)
$$

The Lagrangian density is a density(!), that is, something which can be integrated over $X$. If $X$ is oriented then it can be taken to be a differential $d$-form.

Before reviewing the main properties of an action, let's note how some familiar examples fit into this scheme.

Exercise 1.3. Consider first classical mechanics as a field theory with $d=1$. Look first at a particle moving in $\mathbb{R}^{3}$. Suppose that $X=[0, T]$. Then $\mathcal{C}_{X}$ is the space of paths in $\mathbb{R}^{3}$. If there is no potential, then what is the action? What is the Lagrangian density? What if there is now a potential function $V: \mathbb{R}^{3} \rightarrow \mathbb{R}$ ? What if we replace $\mathbb{R}^{3}$ by an arbitrary Riemannian manifold $M$ ?

Exercise 1.4. A more invariant formulation of classical mechanics is as a field theory on 1 dimensional Riemannian manifolds $X$. Can you formulate the Lagrangian density and action in this case? (The fields should be paths into a fixed Riemannian manifold $M$.) Note that if $X$ is diffeomorphic to an interval, then it is isometric to $[0, T]$ for some $T$. In other words, the only invariant of a Riemannian interval is its length. This is a fancy way of stating parametrization by arclength.

Exercise 1.5. Generalize the previous example to the $\sigma$-model in $d$ dimensions. This is a $d$ dimensional field theory formulated on Riemannian $d$-manifolds. There is a fixed auxiliary Riemannian manifold $M$, and the fields on a $d$ dimensional Riemannian manifold $X$ are smooth maps $\phi: X \rightarrow M$. The Lagrangian density is

$$
L_{X}(\phi)=|d \phi|^{2} d \mu_{X}
$$

where $d \mu_{X}$ is the Riemannian volume density. Write $L_{X}$ in local coordinates, or in a form recognizable to you. Prove that this action is conformally invariant in $d=2$ dimensions.

Exercise 1.6. Here is a simple topological example in $d$ dimensions. Fix a manifold $M$ (no metric!) and a $d$-form $\omega \in \Omega_{X}^{d}$. The spacetimes are now simply oriented $d$-manifolds $X$ and the space of fields $\mathcal{C}_{X}$ is the space of smooth maps $\phi: X \rightarrow M$ (as it is for the $\sigma$-model). The lagrangian density, or better lagrangian form, is $L_{X}(\phi)=\phi^{*} \omega$. Write this in local coordinates. Are there any simplifications if $\omega$ is closed $(d \omega=0)$ ?

Exercise 1.7. Try to formulate free field theories in this formalism. Consider for example a free scalar field or a free spinor field. What is the precise class of spacetimes considered? What is the space of fields? Do you know some interaction terms to add to these Lagrangians?

Exercise 1.8. Consider now a gauge theory. We will be doing this in more detail later, but it is a good idea for you now to think of how this fits in with our formalism. To be concrete, consider Yang-Mills in 4 dimensions. Now for a Riemannian 4-manifold $X$ the space of fields is a space of connections. Can you see how to fit gauge symmetry into the picture? What is the action? Does Yang-Mills make sense in other dimensions? Show that only in $d=4$ is the Yang-Mills lagrangian conformally invariant. Show that in $d=2$ it is invariant under area-preserving diffeomorphisms. (This is crucial in David Gross' lectures.) 
There are two main properties of fields and classical actions I want to mention here: symmetry and locality. I may use the term 'functoriality' for the symmetry I am talking about here, or perhaps 'external symmetry'. It is not like gauge symmetry (Exercise 1.8) which is a symmetry of the fields, something we might call an 'internal symmetry.' Rather, it refers to the symmetries of the spacetimes. Such a symmetry is a diffeomorphism $f: X^{\prime} \rightarrow X$ which preserves all of the structure. So if we are dealing with a Euclidean field theory, the map $f$ is required to preserve the metrics, i.e., $f$ is an isometry. Of course, we might have $X^{\prime}=X$ which is the most interesting case. In any case we require that a symmetry induce a map on fields

$$
f^{*}: \mathcal{C}_{X} \longrightarrow \mathcal{C}_{X^{\prime}}
$$

and that the action be preserved:

$$
S_{X^{\prime}}\left(f^{*} \phi\right)=S_{X}(\phi), \quad \phi \in \mathcal{C}_{X}
$$

If there is a Lagrangian density, then we usually have the stronger condition that the density is preserved, at least up to an exact term.
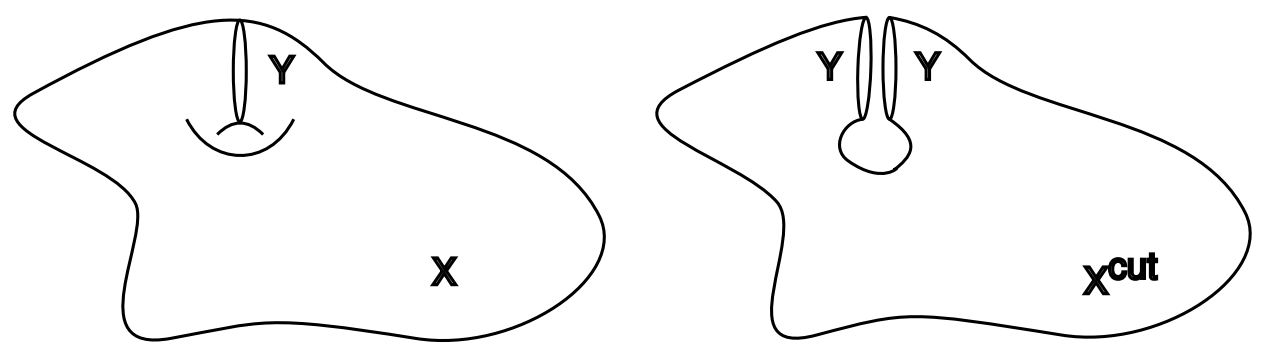

Figure 1: Cutting a spacetime $X$ along $Y$ to obtain $X^{\text {cut }}$

Locality is the assertion that the fields are local objects and that the action can be computed locally. There are many ways to formulate this, and for our purposes we focus on the following situation. Suppose $X$ is a $d$ dimensional spacetime and $Y \hookrightarrow X$ is a closed codimension one submanifold of $X$. If we cut $X$ along $Y$ then we obtain a new spacetime which I will call $X^{\text {cut }}$. Notice that we do not require that our spacetimes be connected, nor that they have connected boundaries. The usual picture is Figure 2, in which $X$ is connected and $X^{\text {cut }}$ has two components. But this is not necessary. Nor is it necessary that $Y$ be connected. In any case note that $X^{\text {cut }}$ has two new pieces in the boundary, each of which is diffeomorphic to $Y$. (One of them appears with the opposite orientation.) The situation is illustrated in Figure 1. Notice that there is a gluing map $g: X^{\text {cut }} \rightarrow X$ which identifies these two boundary components. Suppose now we have a field $\phi$ on $X$. Then there is a pullback field $\phi^{\text {cut }}$ on $X^{\text {cut }}$ and we require that

$$
S_{X^{\text {cut }}}\left(\phi^{\text {cut }}\right)=S_{X}(\phi) .
$$



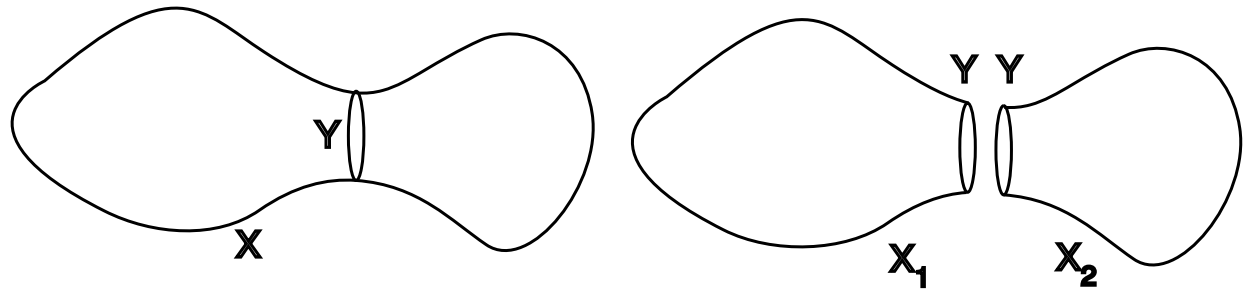

Figure 2: Cutting $X$ into two pieces

This is trivial if the action is given by an integral as in (1.2).

Exercise 1.12. Consider a field theory formulated on Minkowski space. Show that (1.10) is the assertion that the action is invariant under the Poincaré group. How does the Poincaré group act on scalar fields? On spinor fields?

Exercise 1.13. Verify (1.10) and (1.11) for the examples you considered earlier.

Exercise 1.14. If the spacetimes in a field theory have an orientation, then usually the action satisfies:

$$
S_{-X}(\phi)=-S_{X}(\phi)
$$

where $-X$ denotes the manifold $X$ with the opposite orientation. Check this out in examples. On field theories formulated on Minkowski space, what does this say about Lorentz transformations not connected to the identity?

Exercise 1.16. Can you think of more general formulations of locality? What example(s) motivates your formulation?

\section{The Wess-Zumino-Witten Action.}

As motivation for our extension of the notion of the classical action we consider an action which is somewhat more interesting geometrically than those indicated in the exercises above. Often this Wess-Zumino-Witten (WZW) action is just one term in the lagrangian. In $d=2$ it is added to the $\sigma$-model lagrangian (Exercise 1.5) to define one of the fundamental conformally invariant field theories in 2 dimensions. From a mathematician's point of view the corresponding quantum field theory teaches us much about the representation theory of loop groups. In any case, our interest for the moment is in the geometry of the classical action.

Let $G=S U(2)$ be the Lie group of $2 \times 2$ unitary matrices of determinant one. Recall that as a manifold $G$ is diffeomorphic to the 3 -sphere. Let $g$ denote a general element of $G$, and then

$$
\theta=g^{-1} d g
$$

is a matrix-valued differential 1-form on $G$. We are interested in the (scalar) differential 3-form

$$
\omega=c \operatorname{Tr}\left(\theta^{3}\right)
$$


where $c$ is chosen so that

$$
\int_{S U(2)} \omega=1
$$

Exercise 1.19. Parametrize $S U(2)$ by matrices $\left(\begin{array}{c}\alpha \bar{\beta} \\ -\bar{\beta} \bar{\alpha}\end{array}\right)$, where $\alpha, \beta \in \mathbb{C}$ and $|\alpha|^{2}+|\beta|^{2}=1$. Write $\theta$ and $\omega$ in terms of $\alpha$ and $\beta$. Also, compute the constant $c$.

Exercise 1.20. More generally, let $G$ be any compact Lie group. Define the analog of the forms $\theta$ and $\omega$. To do this you will need to pick an invariant inner product on the Lie algebra of $G$. You can carry through the following discussion for this more general case if $G$ is simply connected. If $G$ is not simply connected, then you will need much fancier (but interesting) ideas.

The spacetimes in this $(1+1)$ dimensional field theory are oriented 2-manifolds (surfaces). Let $X$ be a closed oriented surface. Then the fields we are interested in are simply maps to $G$ :

$$
\mathcal{C}_{X}=\operatorname{Map}(X, G) .
$$

Suppose that $\phi: X \rightarrow G$ is such a map. Since $G$ is diffeomorphic to a 3 -sphere we can always extend to a map $\Phi: W \rightarrow G$ where $W$ is an oriented 3 -manifold ${ }^{3}$ whose boundary is $X$, and the restriction of $\Phi$ to the boundary of $W$ is $\phi$. We write $\partial \Phi=\phi$. Now define

$$
S_{X}(\Phi)=\int_{W} \Phi^{*}(\omega)
$$

Provisionally, this is the action, but we want something which only depends on $\phi$, not on the extension $\Phi$. It is straightforward to see that different choices of $\Phi$ change (1.21) by an integer (this requires (1.18)), so that

$$
e^{2 \pi i S_{X}(\phi)}:=e^{2 \pi i S_{X}(\Phi)}
$$

is independent of $\Phi$. This is the (exponentiated) WZW action, and it takes values in the complex numbers. Note that since $\omega$ is real, it always has unit norm.

Exercise 1.23. Verify the assertion that (1.22) is independent of the extension $\Phi$.

Exercise 1.24. Prove that the WZW action is functorial for orientation-preserving diffeomorphisms $f: X^{\prime} \rightarrow X$, in the sense of (1.9) and (1.10).

Exercise 1.25. Prove that the exponentiated WZW action is a smooth function on $\mathcal{C}_{X}$. Compute its differential and find the critical points.

Notice that $S_{X}(\phi)$ is not well-defined, only the exponential $e^{2 \pi i S_{X}(\phi)}$ is. This is fine from the path integral point of view: Typically one writes the integrand of the path integral as $e^{i / \hbar S_{X}(\phi)}$, and we can relax the normalization condition (1.18) and instead require that the "coupling constant" $c$ in (1.17) only take a discrete set of values which makes $e^{i / \hbar S_{X}(\phi)}$ well-defined. This quantization of the coupling

\footnotetext{
${ }^{3}$ Actually, it is better to say that $W$ is an oriented 3-chain in the sense of homology theory.
} 
constant is well-known. Notice also that this action does not have a lagrangian density in the sense of (1.2).

The interesting point is to define the WZW action on an oriented surface $X$ with nontrivial boundary $\partial X$. Thus suppose $\phi: X \rightarrow G$. Since now $\partial X \neq \emptyset$ we can neither write $X$ nor $\phi$ as a boundary, and so we cannot immediately find a 3 chain in $G$ over which to integrate our 3 -form $\omega$. Thus we proceed as follows. The boundary $\partial X$ of $X$ is a disjoint union of circles. Let $\hat{X}=X \cup D$ denote the closed surface obtained from $X$ by gluing discs onto each of the boundary components. The union of those discs is denoted $D .{ }^{4}$ Extend $\phi$ to a map $\phi \cup \Gamma: \hat{X} \rightarrow G$, where $\Gamma$ is the map on the union of the discs. Then the exponentiated action $e^{2 \pi i S_{\hat{X}}(\phi \cup \Gamma)}$ is well-defined. However, we need to investigate its dependence on the arbitrarily chosen $\Gamma$.

Exercise 1.26. Show that if $\Gamma^{\prime}$ is another extension, then

$$
e^{2 \pi i S_{\hat{X}}\left(\phi \cup \Gamma^{\prime}\right)}=e^{2 \pi i S_{-D} \cup D\left(\Gamma \cup \Gamma^{\prime}\right)} \cdot e^{2 \pi i S_{\hat{X}}(\phi \cup \Gamma)},
$$

where $-D \cup D$ is the union of 2 -spheres obtained by gluing $D$ with the opposite orientation to another copy of $D$. (This is usually called the double of $D$.) Now define

$$
c_{D}\left(\Gamma^{\prime}, \Gamma\right)=e^{2 \pi i S_{-D \cup D}\left(\Gamma \cup \Gamma^{\prime}\right)} .
$$

Conclude that for maps $\Gamma, \Gamma^{\prime}, \Gamma^{\prime \prime}: D \rightarrow G$ we have

$$
c_{D}\left(\Gamma^{\prime \prime}, \Gamma\right)=c_{D}\left(\Gamma^{\prime \prime}, \Gamma^{\prime}\right) \cdot c_{D}\left(\Gamma^{\prime}, \Gamma\right)
$$

Now we have a sort of exponentiated action for a field $\phi$ on the surface $X$ with boundary. It is not simply a number, however. It is a (complex-valued) function depending on how we extend $\phi$ to $\hat{X}$, in other words, depending on $\Gamma$. Furthermore, the dependence on $\Gamma$ is fairly simple (1.27), and most importantly the factor (1.28) which arises can be computed purely in terms of $\Gamma, \Gamma^{\prime}$, i.e., it does not depend on $\phi$. (This is really a manifestation of locality.) One might be content to stop here and say that this function of $\Gamma$ is the exponentiated action. However, there is a nicer geometric way to proceed, and this is our reason for considering this example.

Here, then, is our typical mathematician's ploy. We have an example of a function which obeys a certain equation (1.27). So let's consider the set of all functions which satisfy the same equation. To do that we first need to identify the set of allowable $\Gamma$. Let $\gamma=\partial \phi$ be the restriction of $\phi$ to the boundary $\partial X=\partial D$. Then the set of $\Gamma$ is the set of fields on $D$ whose restriction to $\partial D$ is $\gamma$ :

$$
\mathcal{C}_{D}(\gamma)=\{\Gamma: D \rightarrow G: \partial \Gamma=\gamma\}
$$

Now we define the set of functions which satisfy (1.27), giving it the suggestive name $L_{\partial X}(\gamma)$ :

$$
L_{\partial X}(\gamma)=\left\{\ell: \mathcal{C}_{D}(\gamma) \rightarrow \mathbb{C}: \ell\left(\Gamma^{\prime}\right)=c_{D}\left(\Gamma^{\prime}, \Gamma\right) \cdot \ell(\Gamma)\right\}
$$

\footnotetext{
${ }^{4}$ Notice that we allow $\partial X$ to have multiple components. Recall that we do not require that any of our manifolds (for example $X$ ) to be connected.
} 
Notice that this set depends only on $\gamma$, as is indicated by the notation. Now what does $L_{\partial X}(\gamma)$ look like? I claim that it is a one dimensional complex vector space, also known as a complex line. Furthermore, I claim that it has a natural inner product, so is actually a hermitian line.

Exercise 1.30. Prove these last two assertions by constructing the vector space structure and inner product and verifying that $L_{\partial X}(\gamma)$ is one dimensional.

Finally, notice that the definition of $L_{\partial X}(\gamma)$ does not use the fact that $\partial X$ is the boundary of a surface. In other words, for any field ${ }^{5} \gamma \in \mathcal{C}_{Y}$ on a closed oriented 1-manifold ${ }^{6} Y$ we use (1.29) to define a hermitian line $L_{Y}(\gamma)$. (Then $D$ is the manifold obtained by attaching a disk to each component of $Y$.)

So to summarize we have used the 3 -form $\omega$ to define two mappings:

$$
\begin{aligned}
& \phi \in \mathcal{C}_{X} \longmapsto e^{2 \pi i S_{X}(\phi)} \in L_{\partial X}(\partial \phi), \\
& \gamma \in \mathcal{C}_{Y} \longmapsto L_{Y}(\gamma) .
\end{aligned}
$$

Here $X$ is a compact oriented 2-manifold and $Y$ is a closed oriented 1-manifold. In other words, we allow $X$ to have a boundary, but not $Y$. When $X$ is closed the line $L_{\emptyset}$ is simply the "trivial" hermitian line of complex numbers $\mathbb{C}$. The exponentiated action is then defined by (1.22). In case $X$ has boundary (1.31) is a convenient way to look at the construction preceding Exercise 1.26.

Let me say it again: The exponentiated action on a manifold with boundary is not a complex number, but now takes values in a hermitian line. This is already a modification of the scheme we outlined in the previous section, but this action still has the essential locality property — or gluing law_written in (1.11), only here it is expressed in a different form-equation (1.34) of the following exercise.

Exercise 1.33. Let $X$ be a compact oriented 2-manifold, $Y \hookrightarrow X$ a closed codimension one submanifold, and $X^{\text {cut }}$ the manifold obtained by cutting $X$ along $Y$ (Figure 1). Let $\phi \in \mathcal{C}_{X}$ and $\phi^{\text {cut }} \in \mathcal{C}_{X^{\text {cut }}}$ the corresponding field on $X^{\text {cut }}$, as in (1.11). Prove that

$$
\operatorname{Tr}_{Y} e^{2 \pi i S_{X \text { cut }}\left(\phi^{\text {cut }}\right)}=e^{2 \pi i S_{X}(\phi)},
$$

where $\operatorname{Tr}_{Y}$ is performed using the hermitian metric in the line $L_{Y}\left(\left.\phi\right|_{Y}\right)$. You will need to use the properties (1.36) and (1.37) below to make sense of (1.34).

Now we make an even larger extension/modification of the scheme in the previous section, and this is our key point in this section. We consider (1.32) as the definition of an (exponentiated) WZW action for fields on a 1-manifold. This is not at all the usual picture. First of all, we have an action in a $d$ dimensional theory defined for fields in $d-1$ dimensions. Secondly, the value of the action is not a number, but rather a set (more precisely, a hermitian line). I hope that through the course of these lectures you will be convinced that this is a useful extension of the notion

\footnotetext{
${ }^{5}$ You should take note here that although we originally discussed fields in a $d$ dimensional field theory as defined on $d$ dimensional manifolds (spacetimes), we are now extending that notion to consider fields in a $d$ dimensional field theory defined on $d-1$ dimensional manifolds. Here it is clear that $\mathcal{C}_{Y}=\{\gamma: Y \rightarrow G\}$.

${ }^{6}$ This is simply a finite union of circles.
} 
of a classical action. For now, here are some properties which are analogous to properties of the usual classical action. In particular, Exercise 1.38 deals with symmetry. Locality will have to wait for the next lecture. (Think about what this would mean.)

Exercise 1.35. Construct isomorphisms

$$
L_{Y_{1} \sqcup Y_{2}}\left(\gamma_{1} \sqcup \gamma_{2}\right) \longrightarrow L_{Y_{1}}\left(\gamma_{1}\right) \otimes L_{Y_{2}}\left(\gamma_{2}\right)
$$

and

$$
L_{-Y}(\gamma) \longrightarrow \overline{L_{Y}(\gamma)}
$$

Here ' $\sqcup$ ' denotes the disjoint union (that is, the union of two sets with empty intersection) and $\bar{L}$ is the complex conjugate vector space to $L$ (that is, the vector space with the same underlying addition and the complex conjugate scalar multiplication). Notice that these properties are analogous to properties of the usual classical action. (The isomorphism (1.37) is analogous to the equation (1.15). We did not write the analog of (1.36) for the usual action, but you should easily see what it is. In fact, you can view it as a special case of the gluing law where we glue along an empty manifold!) Show that these isomorphisms are actually isometries.

Exercise 1.38. Suppose $f: Y^{\prime} \rightarrow Y$ is an orientation-preserving diffeomorphism of 1-manifolds. Then for any $\gamma \in \mathcal{C}_{Y}$, construct an isometry

$$
f^{*}: L_{Y}(\gamma) \longrightarrow L_{Y^{\prime}}\left(f^{*} \gamma\right)
$$

Notice that (1.39) is the analog of (1.10).

The next exercise is worked out in [F5]. It is a geometric way to pass from the lagrangian picture (classical action) to the hamiltonian picture.

Exercise 1.40. Show that $L_{Y}(\gamma)$ depends smoothly on $\gamma$ in the sense that these lines fit together into a smooth hermitian line bundle $L_{Y} \rightarrow \mathcal{C}_{Y}$. Define a parallel transport on paths in $\mathcal{C}_{Y}$ using the WZW action. Show that this is actually the parallel transport of a connection on $L_{Y}$. Compute the curvature of this connection.

Exercise 1.41. You can carry out the constructions in this section in much more generality. One generalization is to let $G$ be any compact Lie group, though you will have to work harder if $G$ is not simply connected. Another generalization is to work in $d$ dimensions and replace $\omega \in \Omega^{3}(G)$ by a $(d+1)$-form on a manifold $M$. The normalization condition (1.18) should be replaced by the condition that the integral of this form over all $(d+1)$-cycles is an integer. You will also want to make some topological assumptions on $M$ generalizing simple connectivity in the $d=2$ case. So a simpler case is a $d=1$ field theory based on an integral 2 -form $\omega$ on some manifold $M$. In this case we can find a hermitian line bundle $L \rightarrow M$ with a unitary connection whose curvature is $2 \pi i \omega$. Show that the exponentiated classical action in this case is the parallel transport (or holonomy) of this connection, and the action (1.32) just reproduces the line bundle $L$. (This last assertion is not as precise as it might be — what is the precise statement?)

Suppose that $Y=S^{1}$ is the standard circle. Then $\mathcal{C}_{S^{1}}=\operatorname{Map}\left(S^{1}, G\right)=L G$ is the loop group; the multiplication of loops is defined pointwise using the multiplication in $G$. According to Exercise 1.40 the lines $L(\gamma)=L_{S^{1}}(\gamma)$ fit together to form a smooth hermitian line bundle $L \rightarrow L G$ over the loop group. Let $\widehat{L G}$ denote the set of elements of unit norm in $L$; it is a principal circle bundle over $L G$. In the next 
exercise you will show that $\widehat{L G}$ is a central extension of $L G$. This construction of the central extension is originally due to J. Mickelsson [M].

Exercise 1.42. Suppose $\gamma_{1}, \gamma_{2} \in L G$. Construct an isometry

$$
L\left(\gamma_{1}\right) \otimes L\left(\gamma_{2}\right) \longrightarrow L\left(\gamma_{1} \gamma_{2}\right)
$$

This is not trivial - it uses a formula sometimes attributed in the physics literature to Polyakov. Restrict (1.43) to the elements of unit norm to define multiplication in $\widehat{L G}$. Verify that this multiplication is associative and indeed defines a group. Construct a homomorphism $\widehat{L G} \rightarrow L G$ and show that its kernel is isomorphic to the circle group of unit complex numbers. Also, show that the kernel is central in $\widehat{L G}$, that is, elements in the kernel commute with every element in $\widehat{L G}$.

Exercise 1.44. Exercise 1.38 asserts that the action of Diff $S^{1}$ on $L G$ lifts to the line bundle $L \rightarrow$ $L G$. How does the lifted action interact with the isometry (1.43)?

For details on the material in this section, see [F5].

\section{§2: Categories, Finite Groups, and Covering Spaces}

We discuss in general terms what ideas are necessary to extend the notion of classical action further. The indicated extension is crucial in understanding the relationship of quantum groups to 3 dimensional TQFT. Unfortunately it involves the concept of a category, which may be off-putting at first. Category theory has been called "the theory of abstract nonsense." Be that as it may, the notion is useful to us here. We then introduce what is surely the simplest field theory: gauge theory with finite gauge group. The interesting structure is in the space of fields; the action we consider is trivial. In the exercises we indicate a "twisted" version of the theory which has nontrivial action. This theory exists in any dimension, though our main interest later is in the $d=3$ case. Then it is a simple example of Chern-Simons theory, which can be defined for any compact gauge group. We remark that although these theories are analytically simple, they still illustrate some basic properties of gauge theory, especially the role of symmetries and reducible connections.

\section{Going Further.}

What I hope you learned from the WZW example is the following. In a $d$ dimensional field theory we can allow actions which are not of the form (1.1) but rather of the form

$$
e^{2 \pi i S_{X}(\cdot)}: \mathcal{C}_{X} \longrightarrow \mathbb{C}
$$

where $S_{X}(\cdot)$ may not be defined. Furthermore, I challenge you to think of any loss (from a physics point of view) in passing from (1.1) to the exponentiated form (2.1) when $S_{X}(\cdot)$ is defined. (I cannot think of any.) Here $X$ is a closed $d$-manifold. We extend the idea of fields and action to closed $(d-1)$-manifolds $Y$ :

$$
L_{Y}(\cdot): \mathcal{C}_{Y} \longrightarrow \mathcal{L}
$$

where $\mathcal{L}$ is the category of all finite dimensional Hilbert spaces. We will have more to say about that shortly, so please don't panic yet! Finally, if $X$ is a $d$-manifold 
with boundary, the exponentiated action (2.1) has a generalization which we can explain using the following diagram of line bundles:

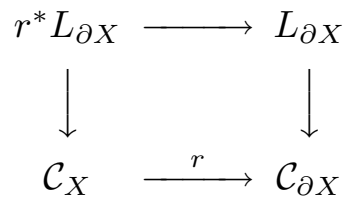

Here $r$ is the restriction map which restricts a field to the boundary. The line bundle $L_{\partial X} \rightarrow \mathcal{C}_{\partial X}$ is the extended action (2.2). Then the action $e^{2 \pi i S_{X}(\cdot)}$ is a section of the line bundle $r^{*} L_{\partial X} \rightarrow \mathcal{C}_{X}$. This extended classical action has several properties, scattered in Lecture 1, which basically capture the idea that the action behaves like the integral over the manifold of something which depends locally on the field. The most characteristic of these properties is the gluing law (1.34).

Exercise 2.4. Perhaps an analogy with honest integration will help understand this idea of an extended action. The usual situation is that we have a compact oriented $d$-manifold $X$ and a $d$ form $\alpha$ on $X$. Then the integral $\int_{X} \alpha$ is defined and is a single number. More generally, consider a fiber bundle $X \rightarrow \mathcal{C}$ whose typical fiber is a compact oriented $d$-manifold, and let $\alpha \in \Omega^{d}(X)$ be a d-form. Then integration over the fibers of $X \rightarrow Y$ produces a function $\int_{X / Y} \alpha$ on $\mathcal{C}$. This is the analog of the usual classical action. But more generally suppose that the fibers of $X \rightarrow \mathcal{C}$ are compact oriented manifolds of dimension $d-i$. Then integration along the fibers gives an $i$-form on the base:

$$
\int_{X / Y} \alpha \in \Omega^{i}(\mathcal{C})
$$

So here we have a family of $(d-i)$-manifolds and we integrate something $d$ dimensional over the fibers to get something $i$ dimensional on the base. If $i=1$ then we get a 1 -form, which you might think of as analogous to a connection form on a hermitian line bundle. This is analogous to our first extension of the classical action, which in this case is not a 1-form but rather a hermitian line bundle.

Incidentally, the exercise here is to fill in the details if you are not already familiar with integration over the fibers. You might also want to consider the analog of Stokes' theorem in this context.

You should now raise several questions. First, how does our extended classical action work in familiar examples? Secondly, are there nontrivial examples other than the WZW action? Finally, can we go further and consider $(d-1)$-manifolds with boundary, closed $(d-2)$-manifolds, etc.? Following the old joke, we will answer these questions in the form of questions! First, the familiar examples.

Exercise 2.5. For the usual examples you considered in the previous section, show that the extended action (1.32) is trivial. However, for usual second order lagrangians the correct space of fields on the boundary should include a derivative. Consider classical mechanics, for example (Exercise 1.4). The space of fields attached to a point $Y=$ pt should be the tangent bundle $T M$, not the manifold $M$. Then the extended action gives a trivial line bundle over $T M$. However the connection constructed following the idea of Exercise 1.40 is nontrivial, and its curvature is the standard symplectic form constructed from the Riemannian metric. Work out the details to the point that you recognize familiar formulas from classical mechanics.

There is another nontrivial example: the Chern-Simons action. I know this best for $d=3$, but in principle it can be worked out in other (odd) dimensions. (The $d=1$ case is Exercise 1.41.) Our main interest in these lectures is the Chern-Simons 
action for a finite gauge group. The case of a continuous gauge group has more geometric interest, and you can find all of the details in [F5]. (There is also an account in [F4].) We consider the finite gauge group case in the next section and defer to these references for the continuous group case.

Consider again a $d$ dimensional field theory. We defined (in an example) an action on $d$-manifolds with boundary which satisfies a gluing law (1.34). Further, we asserted that (1.32) should be considered as an extension of the classical action to closed $(d-1)$-manifolds. Now we want to go further - define an action on $(d-1)$ manifolds with boundary and formulate a gluing law. Let's just see what kind of objects we should expect to run into. Since the action on a closed $(d-1)$-manifold gives a hermitian line, we expect that on a $(d-1)$-manifold with boundary the action is some similar object. (The analogy in $d$ dimensions is that the action on a $d$-manifold with boundary - an element in a complex line - is similar to the action on a closed $d$-manifold - a complex number.) At the very least we expect that it is a set rather than some kind of number. Then the analog of equation (1.34), the gluing law, will be an "equation" between sets. Now such equations are possibleyou can say that two sets are equal - but it is also possible to say that two sets are isomorphic without being equal. This is an extra layer of complexity which sets have that numbers don't. We have already run into this in (1.36), (1.37), (1.39), and (1.43). Another way to put it is that sets have an "internal structure" and it is possible to have automorphisms of this structure. Mathematicians (notably Saunders MacLane [Mc]) have systematized these ideas in the notion of a category. It fits into the progression:

$$
\text { number, set, category. }
$$

A category is a collection of objects and morphisms (maps and arrows) between objects. Two morphisms compose if the second begins where the first ends. The composition is assumed associative, and usually one assumes that there are identity morphisms as well. If we focus on the objects, then the morphisms encode the internal structure that they possess. On the other hand, it is useful to focus on the morphisms as well. For example, a category with one object is simply a set (of morphisms) with an associative composition law and an identity, also known as a semigroup. If a category has more than one object, then we can think of it as a "semigroup with states." The objects represent the states, and in each state there are certain morphisms which are possible. Some change the state and others (automorphisms) do not.

Exercise 2.6. As an example consider the category $\mathcal{V}$ of finite dimensional complex vector spaces and linear maps. So an object in $\mathcal{V}$ is a vector space and a morphism $L: V_{1} \rightarrow V_{2}$ between $V_{1}, V_{2} \in \mathcal{V}$ is a linear map. Note that two vector spaces can be isomorphic but not equal. Also, every vector space of positive dimension has nontrivial automorphisms. You are familiar with the idea that we should consider isomorphic, but distinct, vector spaces as being different. Think of the tangent spaces to the standard 2-sphere. Tangent spaces at different points are isomorphic, but if we could truly think of them as equal (in a "continuous" way) we would quickly construct an everywhere nonzero vector field on the 2 -sphere, which is not possible. Do you know other examples of categories? Other situations in which it is not possible to identify isomorphic objects which are not equal? What if two objects are isomorphic and have no nontrivial automorphisms? Can we safely identify them in that case?

Exercise 2.7. Next we consider maps of categories. (They are usually called functors.) Roughly, a functor maps the objects and morphisms of one category into the objects and morphisms of 
another so that it preserves compositions. As an example, consider the functor $\mathcal{V} \rightarrow \mathcal{V}$ which assigns to each vector space $V \in \mathcal{V}$ its double dual $V^{* *}$. What does this do to morphisms?

Exercise 2.8. The extra layer of structure in a category allows us to define maps between functors, called natural transformations. Suppose that $\mathcal{C}_{1}, \mathcal{C}_{2}$ are categories and $\mathcal{F}_{1}, \mathcal{F}_{2}: \mathcal{C}_{1} \rightarrow \mathcal{C}_{2}$ are functors. Then a natural transformation $\theta: \mathcal{F}_{1} \rightarrow \mathcal{F}_{2}$ is for each object $C \in \mathcal{C}_{1}$ a morphism $\theta(C): \mathcal{F}_{1}(C) \rightarrow \mathcal{F}_{2}(C)$ such that it is compatible with morphisms. This means that for any morphism $C^{\prime} \stackrel{f}{\longrightarrow} C$ in $\mathcal{C}_{1}$ the diagram

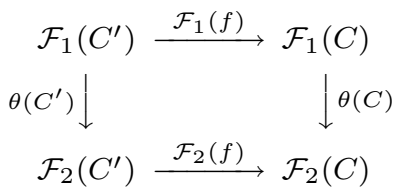

commutes. Construct a natural transformation from the identity functor to the double dual functor of Exercise 2.7. Show that this is in fact a natural isomorphism.

Now we can make an educated guess about going further. The action of a field on a closed $(d-2)$-manifold should be a category, the action of a field on a $(d-1)$-manifold with boundary should take values in the category associated to the boundary field, and there should be a gluing law analogous to (1.34) which is a morphism in a category. We can be even more precise. The type of category where the action takes its values should fit into the progression:

$$
\text { complex number of unit norm, hermitian line, ?. }
$$

Before describing what the '?' is, it is easier to consider the progression of trivial values for the action:

$$
1 \in \mathbb{C}, \quad \mathbb{C}, \quad \mathcal{L} .
$$

In these lectures we only consider a trivial action, so an understanding of (2.10) will suffice. Recall that $\mathcal{L}$ is the category of all finite dimensional Hilbert spaces. A morphism in $\mathcal{L}$ is an isometry. This category is analogous to the complex numbers as the following exercise shows.

Exercise 2.11. We construct a structure on $\mathcal{L}$ analogous to the ring structure on $\mathbb{C}$, that is, the addition and the multiplication. There is a further structure, which is complex conjugation. Then we can build a norm from multiplication and complex conjugation. Construct, then, an addition functor $\mathcal{L} \times \mathcal{L} \rightarrow \mathcal{L}$, a multiplication functor $\mathcal{L} \times \mathcal{L} \rightarrow \mathcal{L}$, and a complex conjugation functor $\mathcal{L} \rightarrow \mathcal{L}$. You should use direct sum, tensor product, and the conjugate linear space. Show that these functors satisfy desired properties, but be careful that where there are equalities in the complex numbers (associativity, commutativity, identity element) there are natural isomorphisms in $\mathcal{L}$. For example, the statement that $\mathbb{C} \in \mathcal{L}$ acts as a multiplicative identity is the assertion that the functor

$$
\begin{aligned}
& \mathcal{L} \longrightarrow \mathcal{L} \\
& L \longmapsto \mathbb{C} \otimes L
\end{aligned}
$$

is naturally isomorphic to the identity functor. So part of the structure on $\mathcal{L}$ is the explicit specification of this natural isomorphism. There is a further layer of structure: equations among these natural isomorphisms. For example, associativity of addition is expressed by a natural isomorphism

$$
\theta_{v_{1}, V_{2}, V_{3}}:\left(V_{1} \otimes V_{2}\right) \otimes V_{3} \longrightarrow V_{1} \otimes\left(V_{2} \otimes V_{3}\right) .
$$


Now for four Hilbert spaces $V_{1}, V_{2}, V_{3}, V_{4}$ there is an equation among the various $\theta$ 's. What is this equation?

In the main flow of these lectures we will not need the general case of '?' in (2.9), only the trivial case in (2.10). I don't mean to make the '?' so mysterious, and I'll give a nontrivial example in the exercises. Perhaps you have already realized that '?' fits into the analogy

$$
\begin{aligned}
& \text { complex numbers : a hermitian line } \\
& \qquad \text { = category of finite dimensionsal Hilbert spaces : ? }
\end{aligned}
$$

We will later introduce the idea of a "2-Hilbert space" and we will see that a '?' is a one dimensional 2-Hilbert space. In any case we now postulate that continuing the progression in (2.1) and (2.2) we have for a closed $(d-2)$-manifold $S$ an assignment

$$
\mathcal{L}_{Y}(\cdot): \mathcal{C}_{S} \longrightarrow \text { collection of one dimensional 2-Hilbert spaces, }
$$

which satisfies properties analogous to those satisfied by (2.1) and (2.2).

Exercise 2.14. Here is a category $\mathcal{K}$ which is a nontrivial example of a one dimensional 2-Hilbert space. Namely, let $\mathcal{K}$ be the category of all unitary representations of the unitary group $U(n)$ which are isomorphic to a direct sum of several copies of the determinant representation on $\mathbb{C}$, where the action of a unitary matrix is multiplication by the determinant. A morphism in $\mathcal{K}$ is an isomorphism of the representations (it commutes with the action of $U(n)$.) Construct a functor $\mathcal{L} \times \mathcal{K} \rightarrow \mathcal{K}$ using the tensor product. This is analogous to scalar multiplication. (Here $\mathcal{L}$ are the scalars.) Construct also the analog of vector addition, namely a functor $\mathcal{K} \times \mathcal{K} \rightarrow \mathcal{K}$, using the direct sum. Show that any irreducible (one dimensional) representation $K_{0} \in \mathcal{K}$ is a "basis" of $\mathcal{K}$. In other words, use $K_{0}$ and scalar multiplication to construct an isomorphism $\mathcal{L} \cong \mathcal{K}$. Consider how this fits with the analogy (2.12). Can you provide a rough definition for a 2-Hilbert space?

Exercise 2.15. State explicitly the properties of (2.13) which are "analogous to those satisfied by (2.1) and (2.2)." Note in particular the gluing law for (2.2):

$$
L_{Y}(\gamma) \cong\left(L_{Y_{1}}\left(\gamma_{1}\right), L_{Y_{2}}\left(\gamma_{2}\right)\right)_{\mathcal{L}_{S}(\partial \gamma)}
$$

Exercise 2.17. Here is an example of a higher dimensional 2-Hilbert space. It will be important in Lecture 4. Let $G$ be a finite group and let $\mathcal{E}$ denote the category of all finite dimensional unitary representations of $G$. Construct an addition $\mathcal{E} \times \mathcal{E} \rightarrow \mathcal{E}$ and a scalar multiplication $\mathcal{L} \times \mathcal{E} \rightarrow \mathcal{E}$. Then construct an inner product $\mathcal{E} \times \mathcal{E} \rightarrow \mathcal{L}$. Can you find an "orthonormal basis" of $\mathcal{E}$ ? What is $\operatorname{dim} \mathcal{E}$ ? This particular example of a 2-Hilbert space is also an algebra. So construct a multiplication $\mathcal{E} \times \mathcal{E} \rightarrow \mathcal{E}$.

Exercise 2.18. Here is an example of a category which is "small" by comparison with the previous examples. It should dispel any illusion you may have that categories are huge. It is also an example which will be crucial for us later. Consider a finite group $G$. Construct a category whose objects are elements of $G$. For each pair of elements $x, g \in G$ we postulate a morphism $g: x \rightarrow g x g^{-1}$. What is composition? Show that if $|G|=n$ this constructs a category with $n$ objects and $n^{2}$ morphisms. Draw a picture for an abelian group. For $G=S_{3}$. 


\section{Finite Gauge Theory.}

Fix a finite group $G$. For example, $G$ could be the cyclic group $\mathbb{Z} / n \mathbb{Z}$ of $n$ elements, or the symmetric group $S_{n}$ of $n$ ! elements. There is no restriction on $G$. We need no other data to define our field theory, though more data is needed for the twisted theory.

Exercise 2.19. For the twisted theory we need to use the classifying space $B G$. One model is the following. Construct an embedding $G \hookrightarrow O(N)$, where $N=|G|$ is the order of $G$. Let $E G$ denote the space of all $N$-tuples of orthonormal vectors in an infinite dimensional dimensional real Hilbert space. Show that $E G$ is contractible and that $O(N)$ acts freely on $E G$. Define $B G$ to the the quotient $E G / G$, where $G$ acts via its embedding into $O(N)$. The space $B G$ has interesting topology, and in the twisted theory we need to fix a cohomology $\operatorname{class}^{7} \lambda \in H^{d}(B G ; \mathbb{R} / \mathbb{Z})$. The untwisted theory in the text corresponds to $\lambda=0$.

The theory we consider is based on "bare" manifolds. No orientation, metric, etc. is needed. Thus a spacetime is simply a compact $d$-manifold $X$ with no additional structure. (The twisted theory is based on oriented manifolds, however.) Symmetries of the spacetimes are simply diffeomorphisms.

Next we define the space of fields on a spacetime $X$. Recall that in our extended notion of field theory we also consider fields on manifolds of dimension less than $d$, so we will define a space of fields for any manifold $M$. Here is the definition:

$$
\mathcal{C}_{M}=\left\{\begin{array}{cc}
P & P \text { is a principal (Galois, regular) } \\
\downarrow & : \quad \text { covering space with structure group } G . \\
M &
\end{array}\right\}
$$

In other terms, a field is a principal bundle $P \rightarrow M$ with structure group $G$. Thus $P$ is a manifold, the group $G$ acts freely on $P$, and the quotient is $P / G=M$. We always take $G$ to act on the right.

As an example, consider $G=\mathbb{Z} / 3 \mathbb{Z}$. If $M=$ pt, then any bundle looks like

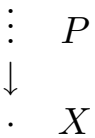

with $G$ cyclically permuting the 3 points. If $M=S^{1}$ then there are 3 possibilities, up to isomorphism, as illustrated in Figure 3. The nontrivial coverings are pictured as pieces of helices, but the endpoints are meant to be identified. Topologically, the total space $P$ in these covers is a circle. The total space of the trivial cover is the disjoint union of 3 circles.

You should have noticed that the space of fields (2.20) is qualitatively different than in the examples in Lecture 1. In usual examples the fields for a smooth manifold (usually infinite dimensional). Here do they not even technically form a set! (The collection of all of anything is not a set-remember Russell!) Rather, they form a category. Warning: Categories enter here in a different way than in our extended notion of classical action in Lecture 1. Remember that categories are a fundamental mathematical structure, just as sets are, and we should not be

\footnotetext{
${ }^{7}$ More precisely, we need to fix a cocycle representing this cohomology class in some model of cohomology.
} 

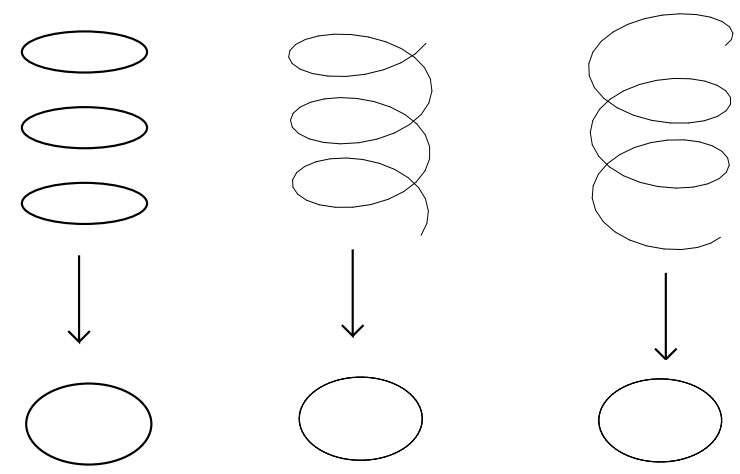

Figure 3: Principal $\mathbb{Z} / 3 \mathbb{Z}$ bundles over $S^{1}$

surprised to see them in a variety of contexts. (We shall meet them again in yet another context later.) What the idea of a category captures here is gauge symmetry. These are symmetries of the fields (internal symmetries), not symmetries of the spacetimes (external symmetries). They are the morphisms in the category of fields $\mathcal{C}_{M}$. Since they are all invertible, we call them 'isomorphisms'.

Definition 2.21. An isomorphism $\varphi$ from $P^{\prime} \in \mathcal{C}_{M}$ to $P \in \mathcal{C}_{M}$ is a diffeomorphism $\varphi: P^{\prime} \rightarrow P$ which commutes with the $G$ action and such that the induced map on the quotient $M$ is the identity.

This means that for $p^{\prime} \in P^{\prime}$ and $g \in G$ we have $\varphi\left(p^{\prime} \cdot g\right)=\varphi\left(p^{\prime}\right) \cdot g$, where the first '.' indicates the $G$ action on $P^{\prime}$ and the second '.' the $G$ action on $P$. Any such map induces a map $\bar{\varphi}: M \rightarrow M$, and we restrict our isomorphism to have $\bar{\varphi}=\mathrm{id}$. More generally, we can consider diagrams

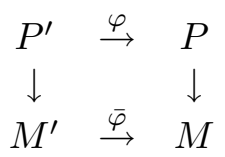

where $\bar{\varphi}$ is possibly nontrivial. They enter when we consider symmetries of the manifolds as well as symmetries of the fields.

Exercise 2.22. Let $f: M^{\prime} \rightarrow M$ be a diffeomorphism. Construct $f^{*}: \mathcal{C}_{M} \rightarrow \mathcal{C}_{M^{\prime}}$ as required by (1.9). In this case it is a functor.

The previous exercise shows how to pull back fields under maps of spacetimes. For these to properly be considered fields we must also be able to cut and paste, as in Figure 1.

Exercise 2.23. Suppose $M$ is a manifold and $N \hookrightarrow M$ is a closed codimension one submanifold of $M$. Let $M^{\text {cut }}$ be the manifold obtained by cutting $M$ along $N$. Let $g: M^{\text {cut }} \rightarrow M$ be the gluing map. Construct a functor

$$
g^{*}: \mathcal{C}_{M} \longrightarrow \mathcal{C}_{M^{\text {cut }}}
$$



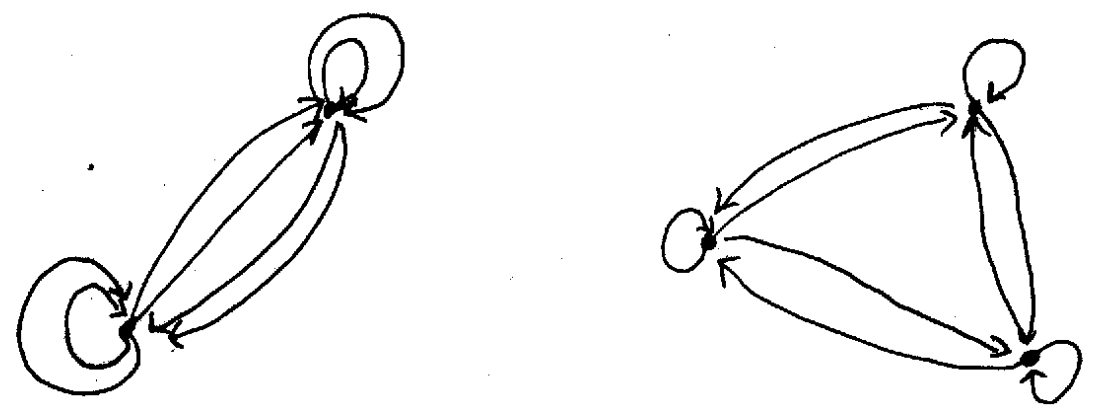

Figure 4: The space of fields $\mathcal{C}_{M}$

So we get a picture of $\mathcal{C}_{M}$ which we schematically render in Figure 4 . Each point is a field and each arrow is a symmetry of fields, that is, an isomorphism. The arrows which start and end at an object $P$ form a group called Aut $P$, the automorphism group of $P$. There is a finite number of arrows from any point to any other. Aut $P$ is also called the group of gauge transformations of $P$. We remark that this picture applies to the space of (gauge) fields in any gauge theory, except that in the general case there are continuous parameters - the space of connections on a fixed bundle - as well as discrete ones - the choice of the bundle. See [F5, $\S 1]$ for a discussion.

The fields $P$ and $P^{\prime}$ are equivalent $\left(P \cong P^{\prime}\right)$ or isomorphic if there is an arrow between them. Let

$$
\overline{\mathcal{C}_{M}}=\text { set of equivalence classes of fields on } M \text {. }
$$

What makes gauge theories with finite gauge group tractable is that $\overline{\mathcal{C}_{M}}$ is a finite set if $M$ is compact.

Exercise 2.24. Determine $\overline{\mathcal{C}_{M}}$ for $M=p t$. For $M=S^{1}$. For $M=[0,1]$.

Exercise 2.25. Show that $\overline{\mathcal{C}_{M}}$ is a finite set for any compact manifold $M$.

Exercise 2.26. I claim that we can not make a field theory where the space of fields associated to $M$ is $\overline{\mathcal{C}_{M}}$. This is because we cannot paste equivalence classes. Consider, for example, cutting the circle $S^{1}$ into an interval [0,1]. Show that we can cut equivalence classes of bundles, but we cannot paste them.

We can determine the space of equivalence classes of fields in terms of the fundamental group. Suppose $M$ is connected. Fix basepoints $m \in M$ and $p \in P_{m}$, where $P_{m}$ is the fiber of $P$ at $m$. Then a field $P \rightarrow M$ determines a map 


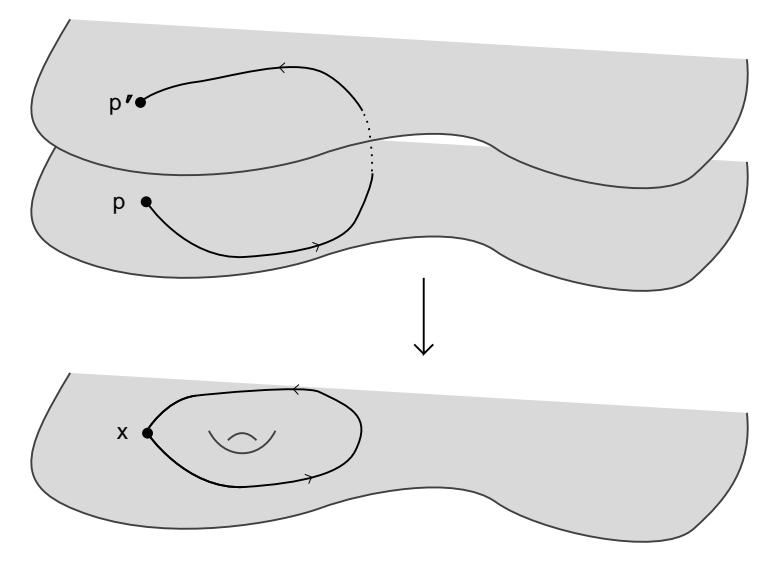

Figure 5: Definition of holonomy

by taking the holonomy around the loop using the basepoint $p$. (See Figure 5.) Any loop at $m$ lifts uniquely to a path in $P$ which starts at $p$ and ends at some $p^{\prime}$ in the fiber $P_{m}$ of $P$ over $m$. The holonomy is the unique $h \in G$ satisfying $p^{\prime}=p \cdot h$. The holonomy only depends on the homotopy class of the loop.

Exercise 2.27.

a) Check this last assertion.

b) Show that the map $\pi_{1}(M, m) \rightarrow G$ defined by holonomy is a homomorphism of groups.

c) If $\gamma$ is this homomorphism, and we change the basepoint $p$ and/or $m$, then the new homomorphism is $g \gamma g^{-1}$ for some $g \in G$.

d) Suppose $M$ is connected and $h: \pi_{1}(M, m) \rightarrow G$ is given. Construct a bundle $P \rightarrow M$ and a basepoint $p \in P_{m}$ such that the holonomy is $h$.

The assertions in Exercise 2.27 hold more generally for any flat connection with arbitrary gauge group. In this case they show that there is an isomorphism (of sets)

$$
\overline{\mathcal{C}_{M}} \cong \operatorname{Hom}\left(\pi_{1}(M), G\right) / G
$$

if $M$ is connected. (We omit the irrelevant basepoint in the notation for the fundamental group.)

Finally we are ready to define the action in this theory. We can summarize in one word: The action is trivial! Recall that we consider a $d$ dimensional theory, so begin with the usual (numerical) action on $d$-manifolds $X$ :

$$
S_{X}(P)=0 \text { for all } P \in \mathcal{C}_{X} .
$$

So this is an example where the usual action (1.1) is defined, not just the exponentiated action (2.1). The extended actions (2.2) and (2.13) are also trivial. So for a closed $(d-1)$-manifold $Y$ we have

$$
L_{Y}(Q)=\mathbb{C} \text { for all } Q \in \mathcal{C}_{Y},
$$

and for a closed $(d-2)$-manifold $S$ we have

$$
\mathcal{L}_{S}(R)=\mathcal{L} \text { for all } R \in \mathcal{C}_{S}
$$


Definitions (2.29) and (2.30) also make sense for manifolds with boundary.

Exercise 2.32. Verify the properties (discussed in Lecture 1) of the extended action - gluing laws, functoriality, etc. The functoriality statement is more involved since one has to worry now about internal symmetries. What is the right formulation?

Exercise 2.33. In the twisted theory the action is nontrivial [FQ], [F1]. We give a brief indication here. Recall that the twisted theory is defined by fixing a cohomology class $\lambda \in H^{d}(B G ; \mathbb{R} / \mathbb{Z})$, or more precisely a cocycle representing the cohomology class. Prove first that for any $G$ bundle $P \rightarrow M$ here exists a "classifying map" $F: P \rightarrow E G$ which commutes with the $G$ action, and that the map is unique up to homotopy through $G$-maps. So the quotient map $\bar{F}: M \rightarrow E G$ is unique up to homotopy. If now $P \in \mathcal{C}_{X}$ for $X$ a closed oriented $d$-manifold, we define

$$
e^{2 \pi i S_{X}(P)}=e^{2 \pi i \bar{F}^{*}(\lambda)[X]}
$$

for any classifying map $F$. Here $[X] \in H_{d}(X)$ is the fundamental class of $X$ given by the orientation. Verify that (2.34) is well-defined.

The twisted version of (2.30) is more complicated. Try to arrive at it much as we arrived at (1.29) in our study of the WZW action. Namely consider first the expression (2.34) for a compact oriented $d$-manifold $X$ with boundary. Now the orientation class is a relative homology class - $[X] \in H_{d}(X, \partial X)$ - and the evaluation $\bar{F}^{*}(\lambda)[X]$ does not make sense. Instead you must choose $d$-cycles on $X$ which represent $[X]$ and evaluate the chosen $d$-cocycle (which represents $\lambda$ ) on such cycles. This depends nontrivially on the choice of the $d$-cycle, and the dependence is encoded in a complex line which just depends on the restriction of $P$ to $\partial X$. Fill in the details and you will have the twisted version of (2.30).

If you are truly ambitious you will carry this further and construct the twisted version of (2.31). This is another motivating example for our extension of the classical action.

Try to formulate (if not prove) the properties of the extended action for this twisted theory. The symmetry properties are perhaps more apparent here since the action is nontrivial. The $d$ dimensional action (twisted (2.29)) is invariant under gauge symmetry, but what is the analogous statement for the $d-1$ dimensional action (twisted (2.30))? What about for the $d-2$ dimensional action (twisted (2.31))?

\section{§3: Generalized Path Integrals}

Now we move from the classical theory to the quantum theory. I dare say we are following Feynman, though we are so far from the original context of his path integrals that I suspect he would be either amused or more likely appalled! Our case is much simpler than usual examples in that the path integral reduces to a finite sum. Nonetheless, the formal picture -rigorous here - is the same in usual examples. We simplify our initial discussion by ignoring the gauge symmetry. The key new idea is an extension of the path integral. We interpret the usual quantum Hilbert space as the result of a path integral over fields on a $(d-1)$-manifold (in a $d$ dimensional theory). Naturally it involves the extended classical action, as does our extension to fields on $(d-2)$-manifolds. We work out the usual path integral and quantum Hilbert space in the finite group gauge theory. In Lecture 4 we will use the extended path integral to compute the quantum group relevant to those theories.

\section{Path Integral Quantization.}

Imagine a $d$ dimensional field theory in which the space of fields $\mathcal{C}_{M}$ attached to any manifold $M$ (of dimension $\leq d$ ) is a finite set. You may as well assume that this is a topological field theory. To simplify the picture even further we assume that there is no (gauge) symmetry among the fields. We put back in the symmetry 
later. However, here we allow a nontrivial action. The partition function of a closed $d$-manifold $X$ is defined to be

$$
Z_{X}=\int_{\mathcal{C}_{X}} e^{2 \pi i S_{X}(P)} d \mu_{X}(P)
$$

We write a typical field as ' $P$ ', keeping in mind our example. The new ingredient is a measure $d \mu_{X}$ on the space of fields $\mathcal{C}_{X}$. With our assumption that $\mathcal{C}_{X}$ is a finite set, the measure is simply a positive number $d \mu_{X}(P)$ for each $P \in \mathcal{C}_{X} \cdot{ }^{8}$. Note that the partition function $Z_{X}$ is a complex number.

Exercise 3.2. Prove that $Z_{X}$ is a topological invariant. That is, if $f: X^{\prime} \rightarrow X$ is a diffeomorphism, then $Z_{X^{\prime}}=Z_{X}$. Remember, we are assuming that this is a topological field theory.

The preceding exercise is the invariance of the partition function under symmetry. It is the quantum analog of (1.10). We now want to investigate the quantum analog of the gluing law (1.11). The following discussion is the usual argument for locality of the path integral via factorization into intermediate states. In our language it goes as follows. Let $X$ be a $d$-manifold, which for simplicity you may assume to be closed, and $Y \hookrightarrow X$ an embedded closed $(d-1)$-manifold. We assume that cutting $X$ along $Y$ splits $X$ into two manifolds $X_{1}$ and $X_{2}$ (see Figure 2). We identify $\partial X_{1}=\partial X_{2}=Y$ The fields fit into the following diagram:

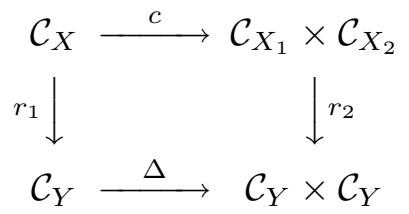

The vertical arrow $r_{1}$ is restriction to $Y$, the arrow $r_{2}$ is restriction to the boundaries of the $X_{i}$, the arrow $\Delta$ is the diagonal inclusion, and $c$ is the pullback under the gluing map. ('c' stands for 'cutting'.) Then we propose to do the integral over $\mathcal{C}_{X}$ in two stages using Fubini's theorem: First integrate over the fibers of $r_{1}$ and then over $\mathcal{C}_{Y}$. Now the gluing law for the classical action (1.34) says that if $P \in \mathcal{C}_{X}$ and $\left\langle P_{1}, P_{2}\right\rangle \in \mathcal{C}_{X_{1}} \times \mathcal{C}_{X_{2}}$ the cut field, then

$$
e^{2 \pi i S_{X}(P)}=\left(e^{2 \pi i S_{X_{1}}\left(P_{1}\right)}, e^{2 \pi i S_{X_{2}}\left(P_{2}\right)}\right)_{L_{Y}(Q)}
$$

where $Q$ is the restriction of $P$ to $Y$. The right hand side is the inner product in

\footnotetext{
${ }^{8}$ Of course, in more typical examples $\mathcal{C}_{X}$ is an infinite dimensional space and a measure $d \mu_{X}$ is extremely difficult to construct and has not been constructed in many examples of interest. Nonetheless, workers in constructive quantum field theory have enjoyed many nontrivial successes in this pursuit.
} 
the line $L_{Y}(Q)$. Carrying out the integration using the Fubini theorem we obtain:

$$
\begin{aligned}
& Z_{X}=\int_{\mathcal{C}_{X}} e^{2 \pi i S_{X}(P)} d \mu_{X}(P) \\
& =\int_{\mathcal{C}_{Y}} \int_{r_{1}^{-1}(Q)} e^{2 \pi i S_{X}(P)} d \mu_{r_{1}^{-1}(Q)}(P) d \mu_{Y}(Q) \\
& =\int_{\mathcal{C}_{Y}} \int_{r_{2}^{-1}(Q, Q)} e^{2 \pi i S_{X}(P)} d \mu_{r_{2}^{-1}(Q, Q)}\left(P^{\text {cut }}\right) d \mu_{Y}(Q) \\
& =\int_{\mathcal{C}_{Y}}\left(\int_{\mathcal{C}_{X_{1}}(Q)} e^{2 \pi i S_{X_{1}}\left(P_{1}\right)} d \mu_{X_{1}}\left(P_{1}\right), \int_{\mathcal{C}_{X_{2}}(Q)} e^{2 \pi i S_{X_{2}}\left(P_{2}\right)} d \mu_{X_{2}}\left(P_{2}\right)\right)_{L_{Y}(Q)} d \mu_{Y}(Q) \text {. }
\end{aligned}
$$

Here we use the definition

$$
\mathcal{C}_{X_{i}}(Q)=\left\{P \in \mathcal{C}_{X_{i}}: \partial P=Q\right\}, \quad Q \in \mathcal{C}_{Y}
$$

Also, we make certain implicit compatibility assumptions about the measure to make this computation.

Exercise 3.5. State explicitly these compatibility assumptions.

To rewrite this last expression in a nicer form, we make the following definitions. Let $X^{\prime}$ be any $d$-manifold with boundary. Then the path integral is a function of a field $Q$ on the boundary:

$$
Z_{X^{\prime}}(Q)=\int_{\mathcal{C}_{X^{\prime}}(Q)} e^{2 \pi i S_{X^{\prime}}(P)} d \mu_{X^{\prime}}(P), \quad Q \in \mathcal{C}_{\partial X^{\prime}}
$$

Note that the integral is over the space of fields with fixed boundary value $Q$. The right hand side of (3.6) takes values in the hermitian line $L_{\partial X^{\prime}}(Q)$, which is the extended action of the field on the boundary (cf. (2.3)). So $Z_{X}$ is a section of the hermitian line bundle $L_{\partial X} \rightarrow \mathcal{C}_{\partial X}$. Again we use the mathematician's ploy of introducing the space of all such sections. Hence for any closed surface $Y$ set

$$
E(Y)=\text { space of sections of the hermitian line bundle } L_{Y} \rightarrow \mathcal{C}_{Y} .
$$

Note that $E(Y)$ is a finite dimensional complex vector space. Then (3.6) determines a relative invariant

$$
Z_{X^{\prime}} \in E\left(\partial X^{\prime}\right)
$$

We impose an $L^{2}$ inner product on (3.7) using a measure $d \mu_{Y}$ on $\mathcal{C}_{Y}$ and the metric on the hermitian line bundle $L_{Y} \rightarrow \mathcal{C}_{Y}$. With these definitions we rewrite (3.4) as:

$$
Z_{X}=\left(Z_{X_{1}}, Z_{X_{2}}\right)_{E(Y)} .
$$

This is the quantum gluing law - the quantum analog of (3.3) - the statement that the path integral is local. 
Thus we have the standard formal ingredients of quantum field theory-the partition function $Z_{X}$ on closed spacetimes and the quantum Hilbert space $E(Y)$ for closed "spaces", i.e., $d$-manifolds. We have indicated that symmetry and locality hold for the partition function. You should now prove that other formal properties of the path integral and quantum Hilbert space follow from the corresponding properties of the classical action. Again you will have to make some assumptions about the measures $d \mu_{X}$ and $d \mu_{Y}$, which I leave you to formulate.

Exercise 3.9. (Functoriality) Show that a diffeomorphism $f: Y^{\prime} \rightarrow Y$ induces an isometry $f_{*}: E\left(Y^{\prime}\right) \rightarrow E(Y)$. In a field theory formulated on Minkowski space this is a representation of the Euclidean group of a space slice on the quantum Hilbert space; the representation of the whole Poincaré group involves the path integral as well. Show that a diffeomorphism $F: X^{\prime} \rightarrow X$ preserves the partition function. (Consider the case where $X$ has nontrivial boundary.)

Exercise 3.10. (Multiplicativity) What can you say about $E\left(Y_{1} \sqcup Y_{2}\right)$ ? What about $Z_{X_{1} \sqcup X_{2}}$ ?

Exercise 3.11. Try this point of view out in some familiar field theories, even those formulated in Minkowski space. In that case, what does the gluing law say about the usual propagation? (Here you should start by considering ordinary quantum mechanics, which is the case $d=1$.) A good nontrivial example is the $d=2$ theory considered in David Gross' lectures: QCD in 2 dimensions with a fixed gauge group. What is the Hilbert space $E\left(S^{1}\right)$ in this theory? Note that this is not a topological theory, but the partition function depends on the area of a surface. What is the correct statement of the gluing law? Of the symmetry properties?

\section{Beyond Quantum Hilbert Spaces.}

You should have noticed that we have not yet considered locality - a gluing law - for the quantum Hilbert space. This is not an idea which is usually explicitly discussed in quantum field theory, though perhaps it is implicit there. For example, consider a discrete system formulated on a lattice in space, i.e., on a $(d-1)$ dimensional lattice $Y$. Then the Hilbert space of the theory is the tensor product over the lattice sites of a finite dimensional Hilbert space $H_{y}$ at each site:

$$
E(Y)=\bigotimes_{y \in Y} H_{y}
$$

Then if the lattice $Y$ is split into two pieces $Y_{1}$ and $Y_{2}$ the Hilbert spaces obviously obey the equation

$$
E(Y) \cong E\left(Y_{1}\right) \otimes E\left(Y_{2}\right)
$$

This is what we mean by saying that the quantum Hilbert space is local, though in general the gluing law is more complicated. Also keep in mind what we did for the classical theory. The classical counterpart to the quantum Hilbert space is the action (2.2), and the gluing law (3.12) is the quantum version of the (trivial case of the) gluing law (2.16).

The Verlinde formulas [V] are a nontrivial example of the locality of the quantum Hilbert space. It was originally formulated for the spaces of "conformal blocks" in 2 dimensional conformal field theory. Then Witten [W] identified these spaces with the quantum Hilbert spaces of 3 dimensional Chern-Simons theory, and in this context the Verlinde formula is a gluing law for the quantum Hilbert spaces. 
It takes the following form. Imagine that a closed surface $Y$ is split into $Y_{1}, Y_{2}$ along a circle $S$. There is a finite number of "labels" $\lambda \in \Lambda$ and for each label a Hilbert space $E\left(Y_{i}\right)(\lambda)$. The Verlinde formula roughly has the form (we ignore some subtleties):

$$
E(Y) \cong \bigoplus_{\lambda \in \Lambda} E\left(Y_{1}\right)(\lambda) \otimes E\left(Y_{2}\right)(\lambda)
$$

Exercise 3.14. Reinterpret this formula along the following lines. Introduce the category $\mathcal{E}$ whose objects are collections of Hilbert spaces indexed by $\Lambda$. Also, introduce the "inner product" $\mathcal{E} \times \mathcal{E} \rightarrow \mathcal{E}$ defined by

$$
\left(\left\{E_{1}(\lambda)\right\},\left\{E_{2}(\lambda)\right\}\right)_{\mathcal{E}}=\bigoplus_{\lambda} E_{1}(\lambda) \otimes E_{2}(\lambda)
$$

Show that this makes $\mathcal{E}$ a 2-Hilbert space (cf. Exercise 2.17). Now rewrite (3.13) in terms of $\mathcal{E}$. Your formula should look like (3.8).

So how should we prove a gluing law for the quantum Hilbert space? The easiest way would be to repeat the computation (3.4). But that requires that we write the quantum Hilbert space as an integral, as in (3.1). This is what we do! It is really the crucial step in these lectures. Recall that our extended classical action (2.2) takes values in hermitian lines. So we write exactly the same equation as (3.1), replacing the classical action $e^{2 \pi i S_{X}(P)}$ by the classical action $L_{Y}(Q)$ for fields on a closed $(d-1)$-manifold:

$$
E(Y)=\int_{\mathcal{C}_{Y}} L_{Y}(Q) d \mu_{Y}(Q)
$$

What does this mean? It is a finite sum

$$
\mu_{1} \cdot L_{1}+\cdots+\mu_{N} \cdot L_{N}
$$

where the $\mu_{i}$ are positive numbers and the $L_{i}$ are hermitian lines. Now we interpret $\mu \cdot L$ as the hermitian line with the same underlying complex vector space as $L$ but with the inner product multiplied by $\mu$. We interpret the sum as the orthogonal direct sum. In this way (3.16) defines a Hilbert space. In fact, it is exactly the same Hilbert space as (3.7).

Exercise 3.17. Verify this last assertion. Recall that we use the $L^{2}$ inner product on (3.7).

Now we repeat the computation (3.4) for a $(d-1)$-manifold $Y$ split along a closed $(d-2)$-manifold $S$. In the course of that we will naturally introduce

$$
\mathcal{E}(S)=\text { space of sections of } \mathcal{L}_{S} \rightarrow \mathcal{C}_{S}
$$

which we could also write as an integral

$$
\mathcal{E}(S)=\int_{\mathcal{C}_{S}} \mathcal{L}_{S}(R) d \mu_{S}(R)
$$


We interpret this in the trivial case where $\mathcal{L}_{S}(R)=\mathcal{L}$, the category of finite dimensional Hilbert spaces. Then an element of $\mathcal{E}(S)$ is a choice of a Hilbert space $W_{R}$ for each field $R \in \mathcal{C}_{S}$. Put differently, an element of $\mathcal{E}(S)$ is simply a hermitian vector bundle over the space of fields $\mathcal{C}_{S}$. So

$$
\mathcal{E}(S)=\operatorname{Vect}\left(\mathcal{C}_{S}\right)
$$

is the collection of such hermitian vector bundles.

You can think of $\mathcal{E}=\mathcal{E}(S)$ as being a "Hilbert space over $\mathcal{L}$ ", analogous to the usual concept of a Hilbert space over $\mathbb{C}$. We call such an object a "2-Hilbert space". (2-vector spaces - the same object without the inner product - were introduced by Kapranov/Voevodsky $[\mathrm{KV}]$ and Lawrence [L].) In Exercise 2.11 we already made the analogy between $\mathbb{C}$ and $\mathcal{L}$. Now go back to Exercise 2.14 and Exercise 2.17 to see what a 2-Hilbert space is. There are operations

$$
\begin{aligned}
+ & : \mathcal{E} \times \mathcal{E} \longrightarrow \mathcal{E} \\
& : \mathcal{L} \times \mathcal{E} \longrightarrow \mathcal{E} \\
(\cdot, \cdot): & \mathcal{E} \times \mathcal{E} \longrightarrow \mathcal{L}
\end{aligned}
$$

analogous to addition, scalar multiplication, and inner product.

Exercise 3.21. Determine the operations (3.20) for the 2-Hilbert space defined in (3.19). What is a basis for this 2-Hilbert space?

Exercise 3.22. State explicitly the symmetry and gluing properties for the quantum integrals (3.1), (3.16), and (3.18). Note in particular the gluing law for the quantum Hilbert spaces:

$$
E(Y)=\left(E\left(Y_{1}\right), E\left(Y_{2}\right)\right)_{\mathcal{E}(S)} .
$$

\begin{tabular}{|c|c|c|}
\hline dimension & classical action & path integral \\
\hline$d \quad(X)$ & $\begin{array}{c}P \longmapsto e^{2 \pi i S_{X}(P)} \\
\text { complex number of unit norm }\end{array}$ & $\begin{array}{c}Z_{X} \\
\text { complex number }\end{array}$ \\
\hline$d-1 \quad(Y)$ & $\begin{array}{l}Q \longmapsto L_{Y}(Q) \\
\text { hermitian line }\end{array}$ & $\begin{array}{c}E(Y) \\
\text { Hilbert space }\end{array}$ \\
\hline$d-2 \quad(S)$ & $\begin{array}{c}\qquad R \longmapsto \mathcal{L}_{S}(R) \\
\text { one dimensional 2-Hilbert space }\end{array}$ & $\begin{array}{c}\mathcal{E}(S) \\
\text { 2-Hilbert space }\end{array}$ \\
\hline
\end{tabular}

How does this fit with (3.12), (3.13), and (3.15)?

We summarize our extended notions of classical action and quantum path integral in the following diagram. Here all of the manifolds are presumed closed for simplicity.

Extended notions of classical action and path integral 


\section{Quantum Finite Gauge Theory.}

We now compute the path integral in finite gauge theory, specializing to $d=3$. In this section we treat the usual path integral and quantum Hilbert space. The new point is to account for the symmetry on the space of fields when carrying out the quantization. In Lecture 4 we will compute the quantum object $\mathcal{E}\left(S^{1}\right)$ associated to the circle and show how it leads to a quantum group.

You should now review the last part of Lecture 2 to be sure you understand the fields and classical action in this theory. (Recall that we consider the "untwisted" case where the classical action is trivial.) We resume that discussion taking over the notation used there. The ingredient we are missing is a measure on the space of fields, which is simply defined:

$$
\mu_{M}(P)=\frac{1}{\# \operatorname{Aut} P}, \quad M \in \mathcal{C}_{M} .
$$

This is the correct "counting measure" and is always how we count objects in mathematics. Symmetries identify equivalent objects which we only want to count once. To be consistent, then, if an object has automorphisms it must be counted as in (3.23).

Exercise 3.24. Verify that this measure is invariant under symmetry.

The partition function is defined by (3.1), except now that we integrate only over the space of equivalence classes of fields $\overline{\mathcal{C}_{X}}$. This makes sense since both the classical action and the measure are invariant under symmetries. Writing ' $\bar{P}$ ' for a typical equivalence class we have

$$
\begin{aligned}
Z_{X} & =\int_{\overline{\mathcal{C}_{X}}} e^{2 \pi i S_{X}(\bar{P})} d \mu_{X}(\bar{P}) \\
& =\sum_{\bar{P}} \frac{1}{\# \operatorname{Aut} P} .
\end{aligned}
$$

Suppose for simplicity that $X$ is connected. Introduce a basepoint $x \in X$ and consider the space of bundles with a basepoint:

$$
\left.\mathcal{C}_{X}^{\prime}=\left\{\begin{array}{c}
P \\
\langle\downarrow \\
X
\end{array}, p\right\rangle: P \text { is a principal } G \text { bundle, } p \in P_{x} \text { a chosen basepoint } .\right\}
$$

Here maps of bundles are required to preserve the basepoint. We need a few facts outlined in the next exercise.

Exercise 3.27. Show that objects in $\mathcal{C}_{X}^{\prime}$ are rigid, that is, have no nontrivial automorphisms. In other words, once we specify an isomorphism of bundles on the basepoint, we know it everywhere. Show that the holonomy sets up a 1:1 correspondence between equivalence classes of elements in $\mathcal{C}_{X}^{\prime}$ and homomorphisms $\pi_{1}(X, x) \rightarrow G$. There is a $G$ action on $\mathcal{C}_{X}^{\prime}$ : A group element $g \in G$ simply moves the pair $\langle P, p\rangle$ to $\langle P, p \cdot g\rangle$. Show that this action passes to the quotient $\mathcal{C}_{X}^{\prime}$. Show that this quotient action corresponds to the action of $G$ by conjugation on the space of homomorphisms $\operatorname{Hom}\left(\pi_{1}(X, x), G\right)$. 
Now the counting measure on $\mathcal{C}_{X}^{\prime}$ weights each bundle with basepoint with weight 1 , since there is only the identity automorphism. Taking into account the $G$ action we find

$$
Z_{X}=\frac{\# \operatorname{Hom}\left(\pi_{1}(X), G\right)}{\# G}
$$

This is obviously a topological invariant of $X$.

We now compute the quantum Hilbert space, defined by (3.7) or (3.16). Let $Y$ be a closed surface. To account for the gauge symmetry we again integrate over the space of equivalence classes of fields. What this means is that we replace (3.7) by the space of invariant sections, that is, sections invariant under gauge transformations. Since the action is trivial (2.30), this is merely the space of invariant functions, i.e., the space of functions on the quotient $\overline{\mathcal{C}_{Y}} \cong \operatorname{Hom}\left(\pi_{1}(Y), G\right) / G$. For this last equality we assume that $Y$ is connected. The $L^{2}$ metric is defined using the measure (3.23). Our argument with basepoints above identifies this with

$$
E(Y) \cong \frac{1}{\# G} \cdot L^{2}\left(\operatorname{Hom}\left(\pi_{1}(Y, y), G\right), G\right)^{G}
$$

Here $y$ is any basepoint in the connected space $Y$, the symbol ' $(\cdot)^{G}$ ' means the invariants under the $G$ action by conjugation, the $L^{2}$ metric weights each homomorphism with unit weight, and the prefactor $1 / \# G$ multiplies this $L^{2}$ metric.

Exercise 3.30. Verify (3.29). What is the answer for $Y=S^{2}$ ? What about $Y=S^{1} \times S^{1}$ ? For this example note that $\operatorname{Hom}\left(\pi_{1}(Y, y), G\right)$ is the set of commuting pairs of elements in $G$. To study the action of conjugation on this set it helps to consider the picture in Exercise 2.18.

Exercise 3.31. By general arguments symmetries of $Y$ are implemented as linear isometries of $E(Y)$. Do some explicit computations for $Y=S^{1} \times S^{1}$. For example, compute the effect of the diffeomorphism defined by the matrix $T=\left(\begin{array}{ll}1 & 1 \\ 0 & 1\end{array}\right) \in S L(2 ; \mathbb{Z})$.

Exercise 3.32. Compute the path integral on a 3-manifold with boundary. Verify the gluing law for this relative invariant.

Exercise 3.33. The $d=2$ case of this theory is also interesting. It is the finite group version of the zero area limit (or topological limit) of 2 dimensional QCD as considered in the lectures of David Gross. Now the partition function is defined for surfaces and there is a basic quantum Hilbert space $E$ attached to the circle. Show that $E$ can be identified as the space of central functions on $G$, that is, functions on $G$ invariant under conjugation. What is the measure? Show that the path integral over a "pair of pants" leads to a multiplication on $E$. What is the multiplication? Can you diagonalize it? Use the gluing law to compute the partition function on any closed surface. Compare with (3.28) to obtain a formula which counts homomorphisms from a surface group into a finite group.

Exercise 3.34. Try to do some computations for a twisted theory (in $d=3$ ) defined by a nonzero $\lambda \in H^{3}(B G ; \mathbb{R} / \mathbb{Z})$. For example, take $G$ to be a cyclic group of order $n$. Then $H^{3}(B G ; \mathbb{R} / \mathbb{Z})$ is also cyclic of order $n$, and we can take $\lambda$ to be the generator. Compute the result for $X$ the projective 3 -space, or more generally a lens space. 


\section{§4: The Quantum Group}

Finally in this lecture we produce the quantum group in finite group ChernSimons theory. For simplicity we only consider the untwisted theory, and not the twisted theory which we indicated in the exercises of Lecture 2. However, we should remark that the precise computations for the twisted theory illustrate some more subtle gluing laws (along codimension 2 submanifolds). The interested reader should consult $[\mathrm{F} 1, \S \S 8-9]$ for details.

Our arguments in this section are somewhat sketchy. A rigorous treatment would at the very least demand that we make precise all of the axioms for 2-Hilbert spaces, and this is already a complicated matter. The reader may refer to $[\mathrm{F} 1, \S 5, \S 7]$ for another treatment.

\section{The 2-Hilbert Space.}

We now compute the 2-Hilbert space $\mathcal{E}=\mathcal{E}\left(S^{1}\right)$ attached to the standard oriented circle. (We take $S^{1}$ to be the unit circle in the complex numbers with the counterclockwise orientation. We also take $1 \in S^{1}$ as a basepoint.) Recall from (3.18) that this is defined as a (generalized) integral over the space of fields on the circle. So our first job is to understand what that space $\mathcal{C}_{S^{1}}$ looks like. As in (3.26) it is best to start by rigidifying the fields by introducing a basepoint. So we consider $\mathcal{C}_{S^{1}}^{\prime}$, the category of principal $G$ bundles over a circle with a chosen basepoint covering the basepoint in $S^{1}$. (For brevity we call them "pointed principal bundles.") Then by starting at the basepoint $p$ and traversing the circle in the properly oriented direction, we arrive back at a point $p \cdot x$, where $x \in G$ is the holonomy. (See Figure 5.) So the holonomy around $S^{1}$ defines a map

$$
\text { hol: } \mathcal{C}_{S^{1}}^{\prime} \longrightarrow G \text {, }
$$

and it is easy to verify that it is an isomorphism on equivalence classes:

$$
\overline{\mathcal{C}_{S^{1}}^{\prime}} \cong G
$$

Now a change of basepoint $p \rightarrow p \cdot g$ changes the holonomy from $x$ to $g^{-1} x g$. Thus the conjugacy class of the holonomy is independent of the basepoint, and

$$
\overline{\mathcal{C}_{S^{1}}} \cong \text { conjugacy classes in } G \text {, }
$$

which agrees with (2.28).

We know by now that it is not enough to work with equivalence classes. So as at the end of the last lecture we use basepoints to construct a good model of $\mathcal{C}_{S^{1}}$. Namely, introduce the category $\mathcal{C}$ whose objects are elements $x \in G$ and with a morphism $x \stackrel{g}{\rightarrow} g x g^{-1}$ for every pair of elements $\langle x, g\rangle$. (See Exercise 2.18.) Think of the object labeled by $x$ as a pointed principal bundle $P_{x}$ with holonomy $x$, and the morphism labeled by $x \stackrel{g}{\rightarrow} g x g^{-1}$ as the map between the pointed principal bundles $P_{x} \rightarrow P_{g x g^{-1}}$ which takes the basepoint in $P_{x}$ to $g$ times the basepoint in $P_{g x g^{-1}}$. These morphisms are isomorphisms of (unpointed) principal bundles, and represent

all of the possible morphisms. For example, the set of arrows $x \stackrel{g}{\rightarrow} x$ is isomorphic to the centralizer $C_{x}$ of $x$ in $G$. This is precisely the set of automorphisms of $P_{x}$ as a principal bundle. Such arguments show that $\mathcal{C}$ is a good model of $\mathcal{C}_{S^{1}}$.

Of course, $\mathcal{C}$ is simply a picture of the action of $G$ on itself by conjugation. 


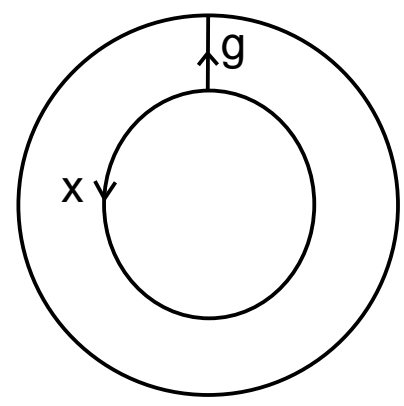

Figure 6: The bundle on $C$ corresponding to $x \stackrel{g}{\rightarrow}$

We can also identify an arrow $x \stackrel{g}{\rightarrow} g x g^{-1}$ as a field on the cylinder $C$. We picture that in Figure 6, where we assume basepoints at the top of each circle and the group element indicates the parallel transport relative to the basepoints. (The cylinder is depicted as an annulus.)

Let $\mathcal{G}$ denote the set of arrows in $\mathcal{C}$; it is a groupoid. (A groupoid is a semigroup in which every arrow is invertible.) For simplicity we denote elements of $\mathcal{G}$ by $x \stackrel{g}{\rightarrow}$. Then the composition law in $\mathcal{G}$ is

$$
\left(x^{\prime} \stackrel{g^{\prime}}{\longrightarrow}\right) \circ(x \stackrel{g}{\longrightarrow})= \begin{cases}x \stackrel{g^{\prime} g}{\longrightarrow}, & x^{\prime}=g x g^{-1} ; \\ \text { undefined, }, & x^{\prime} \neq g x g^{-1} .\end{cases}
$$

We can understand this by gluing two bundles on the cylinder. The inner bundle should correspond to $x \stackrel{g}{\rightarrow}$ in keeping with our convention of representing parallel transport as acting on the left. (It is, after all, a bundle morphism.)

Recall from (2.31) that in this theory the classical action is trivial. (The twisted theory has a nontrivial action.) Now if there were no symmetries and the space of fields were simply $\overline{\mathcal{C}_{S^{1}}}$, which by (4.1) is the set of conjugacy classes in $G$, then by (3.19) we would identify $\mathcal{E}$ with the category of hermitian vector bundles over the set of conjugacy classes in $G$. In other words, an element of $\mathcal{E}$ would be a collection of hermitian vector spaces indexed by the conjugacy classes of $G$. However, this is not correct because of the nontrivial symmetries in the fields.

When we quantize in the top dimension we deal with the symmetries by performing the path integral over the space of equivalence classes of fields (3.25). In the next dimension down, when we quantize the theory on a surface, we accommodate the symmetries by considering invariant sections (3.29). Now we must implement the analogous idea in codimension two: We take "invariant sections" of the trivial bundle whose fiber at each point is $\mathcal{L}$, the category of finite dimensional Hilbert spaces. But rather than consider the large category $\mathcal{C}_{S^{1}}$, we use our model $\mathcal{C}$ for the space of fields on the circle. Then a section is simply a hermitian vector bundle $W \rightarrow G$. In other words, it is a collection of hermitian vector spaces $W_{x}$ indexed by $x \in G$. What do we mean by an "invariant" section? Well, we mean that it should be invariant under the morphisms in $\mathcal{C}$, that is under the action of $G$ on itself by conjugation. However, we do not simply mean that $W_{x}=W_{g x g^{-1}}$. Rather, we mean that we are given an explicit isomorphism

$$
A_{g}: W_{x} \longrightarrow W_{g x g^{-1}}
$$


for each $x, g \in G$. This is in line with the idea that sets have an additional layer of structure: Vector spaces can be isomorphic without being equal. Of course, we presume that the isomorphisms (4.3) compose in accordance with the composition law (4.2) for the arrows. So an element of $\mathcal{E}$ is simply a vector bundle $W \rightarrow G$ together with a lift of the conjugation action of $G$ on itself. The collection of these equivariant vector bundles is usually denoted $\operatorname{Vect}_{G}(G)$. Observe that the dimension of the fiber $W_{x}$, though constant in a conjugacy class, can vary over the group $G$. Also, the bundles we consider have a hermitian structure.

There is one other ingredient in the definition (3.18) of $\mathcal{E}$, namely the measure on the space of fields. The correct measure to use on unpointed bundles is (3.23). However, our pointed bundles are rigid; they don't have any nontrivial automorphisms. But since we have to "divide" by the action of $G$ (by taking invariant sections) we should use the weight $1 / \# G$. (Precisely the same factor occurs when we quantize a closed surface (3.29).) So finally,

$$
\mathcal{E} \cong \frac{1}{\# G} \cdot \operatorname{Vect}_{G}(G)
$$

The standard inner product in $\mathcal{L}$ is $\left(V_{1}, V_{2}\right)=V_{1} \otimes \overline{V_{2}}$, and that in $\operatorname{Vect}(G)$ is obtained by summing over the fibers. To account for the $G$ action we need to take the invariants. Putting this together we see that the inner product in $\mathcal{E}$ is

$$
\left(W_{1}, W_{2}\right)_{\mathcal{E}}=\frac{1}{\# G} \cdot\left(\bigoplus_{x}\left(W_{1}\right)_{x} \otimes \overline{\left(W_{2}\right)_{x}}\right)^{G} .
$$

Note that the right hand side is a finite dimensional Hilbert space, as it should be.

Exercise 4.5. Make explicit the 2-Hilbert space structure of $\mathcal{E}$. What is the addition? Scalar multiplication?

Exercise 4.6. Rewrite (4.4) as a sum over conjugacy classes.

Exercise 4.7. Compute $\mathcal{E}$ for an abelian group $G$.

Next, we observe that $\mathcal{E}$ can be identified as the category of representations of the groupoid $\mathcal{G}$. (In Exercise 2.17 we indicate how the category of representations of a finite group is a 2-Hilbert space. Here we replace a finite group by a finite groupoid.) Namely, associate to an element $W \in \operatorname{Vect}_{G}(G)$ the finite dimensional Hilbert space

$$
W=\bigoplus_{x} W_{x}
$$

(The overloaded notation should not cause confusion.) Then the arrow $(x \stackrel{g}{\rightarrow})$ acts on $W$ by $\left.A_{g}\right|_{W_{x}}$; the action is trivial on $W_{x^{\prime}}$ for $x^{\prime} \neq x$. We can recover the fibers of the vector bundle by $W_{x}=(x \stackrel{e}{\rightarrow})(W)$, where $e$ is the identity element of $G$.

We reformulate this by introducing an algebra $H$ which we might call the "groupoid algebra," by analogy with group algebras:

$$
H=\bigoplus_{x, g} \mathbb{C}\langle x, g\rangle
$$


(We use the symbol ' $\langle x, g\rangle$ ' for an element of $H$ to distinguish it from the corresponding element $(x \stackrel{g}{\rightarrow})$ in $\mathcal{G}$.) So an element of $H$ is a formal linear combination of the symbols $\langle x, g\rangle$ with complex coefficients. The multiplication in $H$ is

$$
\left\langle x_{2}, g_{2}\right\rangle \cdot\left\langle x_{1}, g_{1}\right\rangle= \begin{cases}\left\langle x_{1}, g_{2} g_{1}\right\rangle, & x_{2}=g_{1} x_{1} g_{1}^{-1} \\ 0, & \text { otherwise. }\end{cases}
$$

The unit element is

$$
1=\sum_{x}\langle x, e\rangle
$$

Then $\mathcal{E}$ is the 2-Hilbert space of unitary representations of the algebra $H$, except that the usual inner product is divided by $\# G$.

We pass freely among these various descriptions of $\mathcal{E}$.

What is a basis for $\mathcal{E}$, thought of as a 2-Hilbert space? The geometric picture of equivariant vector bundles $W \rightarrow G$ is perhaps easiest to consider. Note that the fiber $W_{x}$ is a representation of the centralizer $C_{x}$, and that the representations $W_{x^{\prime}}$ and $W_{x}$ are isomorphic if $x$ and $x^{\prime}$ are conjugate. So an indecomposable element of $\operatorname{Vect}_{G}(G)$ is supported on a single conjugacy class and for each $x$ in that conjugacy class is an irreducible representation of the centralizer $C_{x}$. Let $\left\{W_{\lambda}\right\}$ be a set of inequivalent irreducible elements. Then it is a basis for $\mathcal{E}$ in the sense that any other element is isomorphic to $\oplus_{\lambda}\left(V^{\lambda} \otimes W_{\lambda}\right)$ for some finite dimensional Hilbert spaces $V^{\lambda}$ (thought of as trivial vector bundles over $G$ ).

Exercise 4.10. Write a basis of $\mathcal{E}$ for $G$ an abelian group. For $G=S_{3}$, the symmetric group on 3 letters.

\section{Locality and Gluing.}

So far we have just used the extended notion of quantization and the structure of the space of fields on the circle. Now we want to apply the basic principles of locality and gluing to derive further structure on $\mathcal{E}$. At each stage this can be realized by introducing further structure on $H$. By then end we will have introduced enough structure to make $H$ into a quantum group. We begin by considering the path integral over surfaces with boundary.

If $Y$ is any surface with boundary, then from our general picture in Lecture 3 we know that the path integral $E(Y)$ is an element of $\mathcal{E}(\partial Y)$. Now $\partial Y$ is a union of circles, and we would like to identify each of these circles with the standard circle so that $\mathcal{E}(\partial Y)$ can be identified as a tensor product of copies of $\mathcal{E}=\mathcal{E}\left(S^{1}\right)$. In general we cannot do this without introducing additional data (such as a parametrization of the boundary circles), but we will only need to consider surfaces $Y \subset \mathbb{C}$, that is, disks with subdisks removed. Then the boundary is made up of circles in $\mathbb{C}$, and there is a unique composition of a translation and a dilation which brings each circle to the standard circle. In this way we can identify $E(Y)$ as living in a tensor product of copies of $\mathcal{E}$. We can designate some of the boundary components as 'incoming' and some as 'outgoing' and use the hermitian metric in $\mathcal{E}$ to view

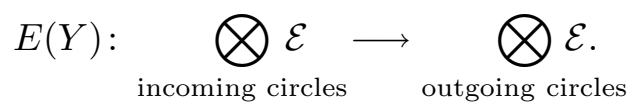


This is a "linear" map of 2-Hilbert spaces, which at the simplest level is a functor between the categories indicated.

Introduce a basepoint on each boundary component of $Y$. Let $\mathcal{C}_{Y}^{\prime}$ denote the category of principal $G$ bundles over $Y$ with chosen basepoints over the basepoints in $\partial Y$. Assume that $Y$ has no closed components. Then arguments similar to those at the end of Lecture 3 show that we can identify

$$
E(Y) \cong L^{2}\left(\overline{\left.\mathcal{C}_{Y}^{\prime}\right)}\right.
$$

Here we think of $\mathcal{E}$ as the 2-Hilbert space of representations of the groupoid $\mathcal{G}$, and the $\mathcal{G}$ action of a given boundary component is computed by gluing a cylinder to that boundary component.

Exercise 4.12. Verify (4.11) in detail.

Exercise 4.13. Suppose that $f: Y^{\prime} \rightarrow Y$ is a diffeomorphism, where $Y, Y^{\prime} \subset \mathbb{C}$ are surfaces as in the previous exercise. Explain how $f$ induces an automorphism of functors $E\left(Y^{\prime}\right) \rightarrow E(Y)$.

Exercise 4.14. Let $C$ denote the cylinder. Compute $E(C)$.

We will compute the path integrals over some surfaces shortly, but we begin by considering symmetries of the circle.

Exercise 4.15. Consider the group of orientation-preserving diffeomorphisms $\mathrm{Diff}^{+}\left(S^{1}\right)$ of the circle. Inside this group is the subgroup of rigid rotations. Construct a retraction of $\mathrm{Diff}^{+}\left(S^{1}\right)$ onto this subgroup. (Hint: Given a diffeomorphism compose with a rotation so that the composition preserves the basepoint. Now cut open the circle at the basepoint; then an orientation-preserving diffeomorphism is a monotonically increasing function $f:[0,1] \rightarrow[0,1]$, and if it preserves the basepoint then $f(0)=0$ and $f(1)=1$.)

Recall from Exercise 3.9 that diffeomorphisms of surfaces induce isometries of the corresponding quantum Hilbert spaces. In Exercise 3.22 you were asked to state the corresponding symmetry, or functoriality, for the 2-Hilbert space associated to a 1-manifold (or a $(d-2)$-manifold in a $d$ dimensional theory). At first glance we might expect a diffeomorphism $f: S^{\prime} \rightarrow S$ to induce an isometry of 2-Hilbert spaces $f_{*}: \mathcal{E}\left(S^{\prime}\right) \rightarrow \mathcal{E}(S)$, and indeed this is true. However, again there is an extra layer of structure: We expect an isotopy $F=f_{t}$ (path of diffeomorphisms) from $f_{0}$ to $f_{1}$ to induce a natural transformation $F_{*}$ from the functor $\left(f_{0}\right)_{*}$ to the functor $\left(f_{1}\right)_{*}$. In topological theories we expect that deformations will not change this natural transformation. Applied to $f_{0}=f_{1}=i d_{S}$, we obtain an action of $\pi_{1}\left(\operatorname{Diff}^{+}(S)\right)$ by automorphisms of the identity functor. Such an automorphism of the identity commutes with all arrows in the category $\mathcal{E}$. More explicitly, an automorphism of the identity specifies for each $W \in \mathcal{E}$ a map

$$
\theta_{W}: W \longrightarrow W
$$

which commutes with all arrows in $\mathcal{E}$.

Exercise 4.16. Let $\operatorname{Rep}(H)$ denote the 2-Hilbert space of representations of a finite group $H$. (See Exercise 2.17.) Determine all automorphisms of the identity of $\operatorname{Rep}(H)$. 
We apply this to the circle. From the previous exercise we know that $\operatorname{Diff}^{+}\left(S^{1}\right)$ is homotopy equivalent to a circle, so the fundamental group is infinite cyclic. It is generated by a circle of rotations. Write an element of the circle as $e^{2 \pi i x}$; then the circle of rotations is

$$
f_{t}\left(e^{2 \pi i x}\right)=e^{2 \pi i(t+x)}, \quad 0 \leq t \leq 1 .
$$

We view this circle of rotations as a diffeomorphism $\tau$ of the cylinder $C$ which is the identity on both boundary circles. We compute the corresponding automorphism of the identity by computing the action on $E(C)$. (See Exercise 4.13.) Now since the cylinder glued to itself is the cylinder, it follows easily that $E(C)$ is the identity map $\mathcal{E} \rightarrow \mathcal{E}$ (Exercise 4.14). We first compute the induced action on fields on the cylinder as

$$
\tau^{*}\langle x, g\rangle=\langle x, g x\rangle=\langle x, g\rangle \cdot\langle x, x\rangle .
$$

Here we use the multiplication in the algebra $H$ (4.8). So the action on a field with holonomy $x$ may be described by gluing on the field $\langle x, x\rangle$. Then Exercise 4.12 implies that the effect on the quantization is the operator $A_{x}: W_{x} \rightarrow W_{x}$, i.e., the automorphism of the identity is

$$
\left.\theta_{W}\right|_{W_{x}}=A_{x}: W_{x} \longrightarrow W_{x} .
$$

In terms of the $H$ action we can describe it as the action of the special element

$$
v=\sum_{x}\langle x, x\rangle
$$

This special element is the inverse of the ribbon element defined by Reshetikhin and Turaev [RT2].

Exercise 4.19. Show that (4.17) induces a diffeomorphism of the torus. In fact, it is the diffeomorphism considered in Exercise 3.31. Compare the automorphism of the identity computed here with the action of that diffeomorphism on the vector space associated to the torus. Is there a gluing law which compares them?

Purely abstract considerations (Schur's lemma) show that an automorphism of the identity of $\mathcal{E}$ acts as multiplication by a scalar on an irreducible element $W_{\lambda}$. We can also see that since $x$ is a central element of the centralizer group $C_{x}$, so acts as a scalar in any irreducible representation. These scalars are the conformal weights of the theory.

Exercise 4.20. Fix an element $x$ and a representation $\lambda$ of the centralizer $C_{x}$. This determines a basis element of $\mathcal{E}$. Compute the conformal weight in terms of the character of $\lambda$.

Exercise 4.21. Calculate the conformal weights explicitly for a cyclic group. For the symmetric group $S_{3}$.

The circle also has an orientation-reversing diffeomorphism - a reflection - which is unique up to isotopy. We expect it to induce a map $\mathcal{E} \rightarrow \overline{\mathcal{E}}$ whose square is the 
identity (since a reflection squares to the identity). Now $\overline{\mathcal{E}}$ means the conjugate 2-Hilbert space, but as a map of categories we can ignore the conjugation - that only affects the "scalar" multiplication. Again we can compute the induced action on $\mathcal{E}$ from the cylinder. Let $\rho$ denote a fixed reflection. Then the induced map on fields is

$$
\rho^{*}\langle x, g\rangle=\left\langle x^{-1}, g\right\rangle \text {. }
$$

Note however that the incoming and outgoing circles are exchanged. This means that an element $W \in \mathcal{E}$ is taken to a new element $W^{*}$ with

$$
\begin{aligned}
\left(W^{*}\right)_{x} & =W_{x^{-1}}^{*} \\
A_{g}^{W^{*}} & =\left(A_{g^{-1}}^{W}\right)^{*} .
\end{aligned}
$$

In terms of the algebra $H$ the duality is implemented by the antipode

$$
S(\langle x, g\rangle)=\left\langle g x^{-1} g^{-1}, g^{-1}\right\rangle \text {. }
$$

Exercise 4.23. In the diffeomorphism group of the circle, write the equation which relates a rotation and a reflection. In the quantization this is reflected by a relation among conformal weights. Namely, show that

$$
\theta_{W^{*}}=\theta_{W}^{*} .
$$

What does this say about conformal weights in terms of our basis?

Next, we consider the path integral over some simple surfaces $Y \subset \mathbb{C}$. For example, consider the unit disk $D$. Since the disk is contractible, all $G$ bundles over $D$ are trivial, as any two basepoints are related by an automorphism we find $\overline{\mathcal{C}_{D}^{\prime}}$ consists of a unique element. It follows that $E(D) \cong L^{2}\left(\overline{\mathcal{C}_{D}^{\prime}}\right)$ is one dimensional and is described by

$$
E(D)_{e}= \begin{cases}\mathbb{C}, & x=e \\ 0, & x \neq e\end{cases}
$$

with $C_{e}=\Gamma$ acting trivially on $E(D)_{e}$. In terms of the algebra $H$ this particular representation is described by a counit $\epsilon: H \rightarrow \mathbb{C}$. From (4.24) we see that the counit is

$$
\epsilon(\langle x, g\rangle)= \begin{cases}1, & x=e ; \\ 0, & \text { otherwise. }\end{cases}
$$

More interesting is the path integral over the "pair of pants," which we realize embedded in $\mathbb{C}$ as the surface $P$ depicted in Figure 7. We view the inside circles as incoming and the outside circle as outgoing (note the orientations), so that $E(P) \in \mathcal{E} \otimes \mathcal{E} \otimes \mathcal{E}$ induces a map

$$
\odot: \mathcal{E} \otimes \mathcal{E} \longrightarrow \mathcal{E}
$$

Here we denote the result of this multiplication map on $W_{1}, W_{2}$ as $W_{1} \odot W_{2}$. There is an induced map on morphisms in $W_{i}$ as well: $\odot$ is a functor! (In category language it gives a "monoidal" structure to $\mathcal{E}$.) 


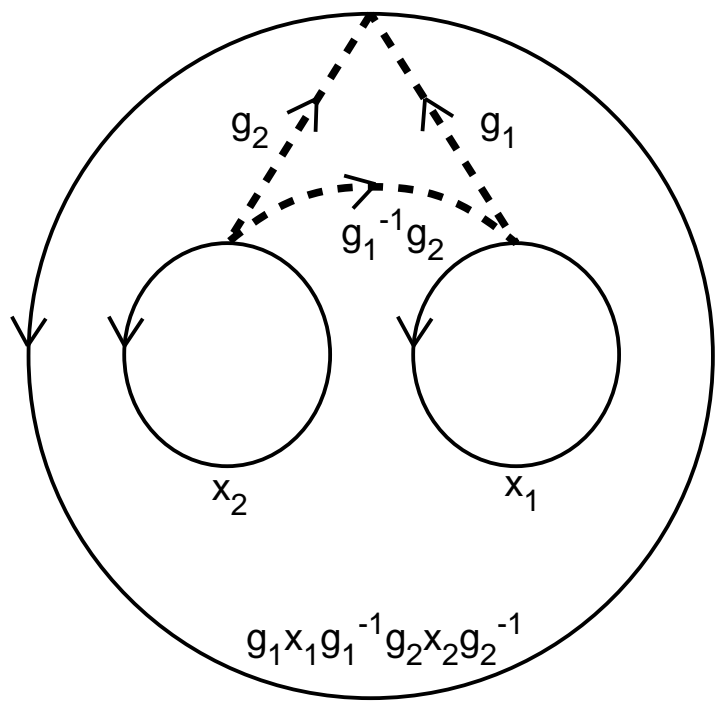

Figure 7: The bundle over $P$ corresponding to $\left\langle x_{1}, g_{1}\right\rangle \times\left\langle x_{2}, g_{2}\right\rangle \in \mathcal{G} \times \mathcal{G}$
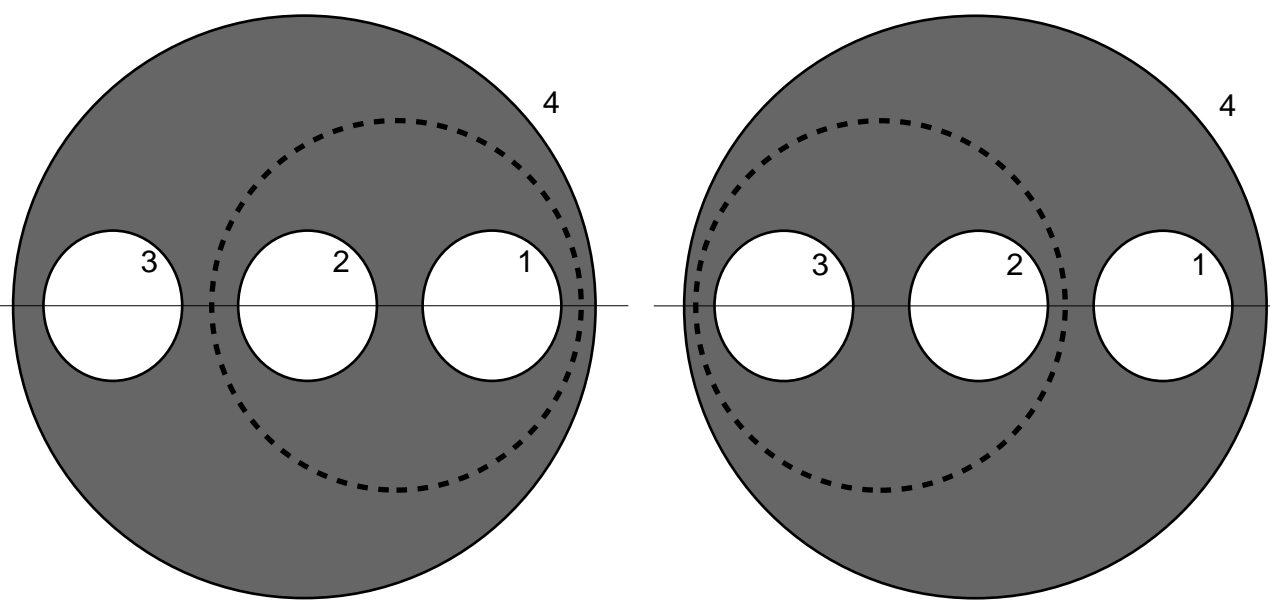

Figure 8: Associativity

Exercise 4.26. Use the gluing law to describe an associativity isometry

$$
\varphi_{W_{1}, W_{2}, W_{3}}:\left(W_{1} \odot W_{2}\right) \odot W_{3} \longrightarrow W_{1} \odot\left(W_{2} \odot W_{3}\right)
$$

(See Figure 8.) In fact, you will find it to be trivial. However, in other theories (e.g. the twisted version of this theory) it is nontrivial.

Exercise 4.27. Use gluing to show that multiplication by (4.24) is isomorphic to the identity map. How is this expressed precisely?

To compute $E(P)$ we need to determine the space of fields on $P$. Following our procedure, we introduce basepoints on each boundary component. 
Exercise 4.28. Show that there is a 1:1 correspondence between $\overline{\mathcal{C}_{P}^{\prime}}$ and $\mathcal{G} \times \mathcal{G}$. Hint: See Figure 7 . In that figure the group elements indicate the parallel transport along the given line relative to the chosen basepoints.

Now we must compute the three different $\mathcal{G}$ actions on $E(P) \cong L^{2}\left(\overline{\mathcal{C}_{P}^{\prime}}\right)$ corresponding to the three boundary circles of $P$. For the inner two boundary circles it is not difficult to verify that the action is by right multiplication. Namely, the action of $\langle x, g\rangle \in \mathcal{G}$ on a function $f(\cdot, \cdot)$ on $\overline{\mathcal{C}_{P}^{\prime}} \cong \mathcal{G} \times \mathcal{G}$ is:

$$
\begin{aligned}
& f(\cdot, \cdot) \longmapsto f\left(\cdot\langle x, g\rangle^{-1}, \cdot\right), \\
& f(\cdot, \cdot) \longmapsto f\left(\cdot, \cdot\langle x, g\rangle^{-1}\right) .
\end{aligned}
$$

On the other hand, the action corresponding to the outer component is

$$
(\langle x, g\rangle \cdot f)\left(\left\langle x_{1}, g_{1}\right\rangle,\left\langle x_{2}, g_{2}\right\rangle\right)= \begin{cases}f\left(\left\langle x_{1}, g g_{1}\right\rangle,\left\langle x_{2}, g g_{2}\right\rangle\right), & \text { if } x=g_{1} x_{1} g_{1}^{-1} g_{2} x_{2} g_{2}^{-1} \\ 0, & \text { otherwise }\end{cases}
$$

Exercise 4.31. Verify (4.29) and (4.30). Recall that in each case the action is induced by gluing on a cylinder to the appropriate boundary component.

To compute $W_{1} \odot W_{2}$, we use the inner product (4.4) in $\mathcal{E}$ to contract the first two factors (corresponding to the inner circles) in $E(P) \in \mathcal{E} \otimes \mathcal{E} \otimes \mathcal{E}$ with $W_{1} \otimes W_{2}$. Up to a factor in the inner product, this says that $W_{1} \odot W_{2}$ is the $\mathcal{G} \times \mathcal{G}$ invariants in $W_{1} \otimes W_{2} \otimes E(P)$. It is not difficult to verify that

$$
\left(W_{1} \odot W_{2}\right)_{x}=\bigoplus_{x_{1} x_{2}=x}\left(W_{1}\right)_{x_{1}} \otimes\left(W_{2}\right)_{x_{2}}
$$

with $G$ action given by

$$
A_{g}^{W_{1} \odot W_{2}}=A_{g}^{W_{1}} \otimes A_{g}^{W_{2}}
$$

Exercise 4.34. Verify (4.32)-(4.33). Also, show that the multiplication $\odot$ can be described by the following operation on $\operatorname{Vect}_{G}(G)$. Namely, let $\mu: G \times G \rightarrow G$ denote multiplication in the group $G$. Then given $W_{1}, W_{2} \in \operatorname{Vect}_{G}(G)$, we can form their external tensor product $W_{1} \otimes W_{2} \rightarrow G \times G$. The quantum product is

$$
W_{1} \odot W_{2}=\mu_{*}\left(W_{1} \otimes W_{2}\right),
$$

where $\mu_{*}$ is the pushforward of vector bundles. (This is defined for finite covering maps.)

Viewing elements of $\mathcal{E}$ as representations of the algebra $H$, a tensor product on representations is induced by a coproduct $\Delta: H \rightarrow H \otimes H$, since then the element $h \in H$ acts in the tensor product $W_{1} \otimes W_{2}$ by $\Delta(h)$. From (4.32) and (4.33) we see that the appropriate coproduct is

$$
\Delta(\langle x, g\rangle)=\sum_{x_{1} x_{2}=x}\left\langle x_{1}, g\right\rangle \otimes\left\langle x_{2}, g\right\rangle .
$$

We content ourselves with one more computation, that of the $R$-matrix. Namely the pair of pants $P$ has a braiding diffeomorphism $\beta$ which acts on the quantization $E(P)$. We depict the braiding in Figure 9. The diffeomorphism $\beta$ rotates 
circle 1 under circle 2, exchanging their positions. It is the identity on circle 3 . Now by Exercise $4.13 \beta$ induces an automorphism of the multiplication $\odot$. Because two of the boundary circles are switched, this means that for each $W_{1}, W_{2} \in \mathcal{E}$ we have a map

$$
R_{W_{1}, W_{2}}: W_{1} \odot W_{2} \longrightarrow W_{2} \odot W_{1}
$$

which commutes with all of the arrows in $\mathcal{E}$. Before proceeding to compute it, we note one property which follows from the structure of $\operatorname{Diff}^{+}(P)$.
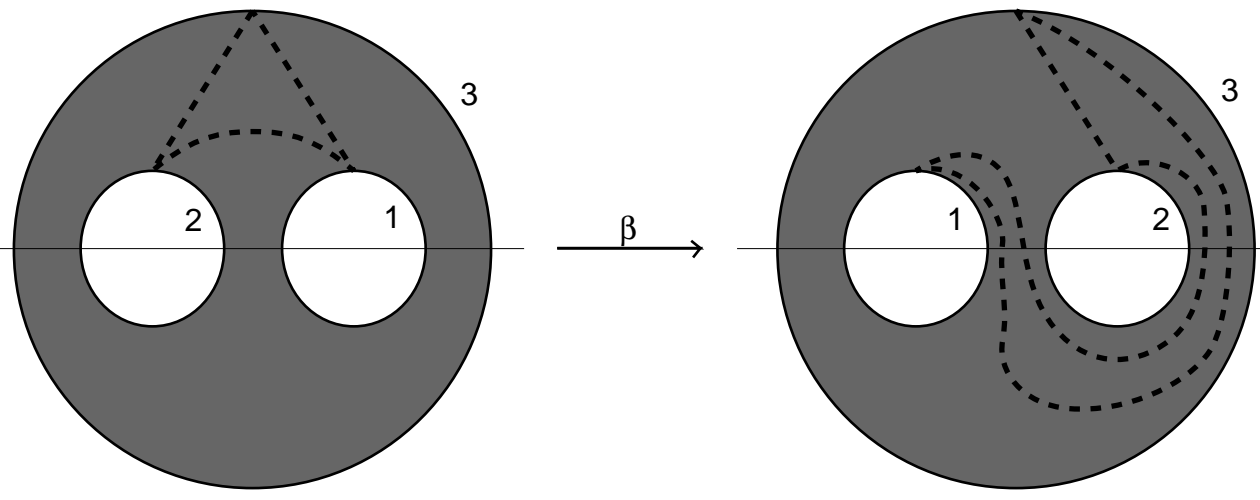

Figure 9: The braiding diffeomorphism $\beta$

Exercise 4.36. Use pictures like Figure 9 to prove that

$$
\tau_{2} \tau_{1} \beta=\beta^{-1} \tau_{3},
$$

where $\tau_{i}$ is a positive Dehn twist around the boundary labeled $i$. Show that this implies that for all $W_{1}, W_{2} \in \mathcal{E}$ the following diagram commutes:

$$
\begin{array}{cc}
W_{1} \odot W_{2} \stackrel{R_{W_{1}, W_{2}}}{\longrightarrow} W_{2} \odot W_{1} \\
\theta_{W_{1} \odot W_{2} \downarrow} \downarrow \theta_{W_{2}} \odot \theta_{W_{1}} \\
W_{1} \odot W_{2} \stackrel{R_{W_{2}, W_{1}}^{-1}}{\longrightarrow} W_{2} \odot W_{1}
\end{array}
$$

Now to the computation of $R_{W_{1}, W_{2}}$. As in all computations the idea is to first see the induced action on the fields. This is easily computed to be

$$
\left\langle x_{1}, g_{1}\right\rangle \times\left\langle x_{2}, g_{2}\right\rangle \stackrel{\beta_{*}}{\longrightarrow}\left\langle x_{2}, g_{1} x_{1} g_{1}^{-1} g_{2}\right\rangle \times\left\langle x_{1}, g_{1}\right\rangle .
$$

Thus on functions $f \in L^{2}\left(\overline{\mathcal{C}_{P}^{\prime}}\right) \cong E(P)$ the induced pushforward action is:

$$
\left(\beta_{*} f\right)\left(\left\langle x_{1}, g_{1}\right\rangle,\left\langle x_{2}, g_{2}\right\rangle\right)=f\left(\left\langle x_{2}, g_{1} x_{1} g_{1}^{-1} g_{2}\right\rangle,\left\langle x_{1}, g_{1}\right\rangle\right) .
$$

Now we must translate this into an action on $W_{1} \odot W_{2}$ using the construction preceding (4.32). The result is

$$
\begin{aligned}
R_{W_{1}, W_{2}}:\left(W_{1}\right)_{x_{1}} \otimes\left(W_{2}\right)_{x_{2}} & \longrightarrow\left(W_{2}\right)_{x_{1} x_{2} x_{1}^{-1}} \otimes\left(W_{1}\right)_{x_{1}} \\
w_{1} \otimes w_{2} & \longmapsto A_{x_{1}}^{W_{2}}\left(w_{2}\right) \otimes w_{1}
\end{aligned}
$$


Exercise 4.38. Verify (4.37).

Finally, this can be implemented universally by a quasitriangular element $R \in$ $H \otimes H$ which satisfies

$$
R_{W_{1}, W_{2}}=\tau_{W_{1}, W_{2}} \circ\left(\rho_{1} \otimes \rho_{2}\right)(R),
$$

where $\tau_{W_{1}, W_{2}}: W_{1} \otimes W_{2} \rightarrow W_{2} \otimes W_{1}$ is the transposition and $\rho_{i}$ are representations of $H$. From (4.37) we deduce

$$
R=\sum_{x_{1}, x_{2}}\left\langle x_{1}, e\right\rangle \otimes\left\langle x_{2}, x_{1}\right\rangle
$$

Thus we have arrived at our goal. Namely from the quantization $\mathcal{E}$ of the circle, using symmetry and gluing laws, we have constructed an algebra $H$ with unit (4.9), counit (4.25), antipode (4.22), comultiplication (4.35), a quasitriangular element (4.39), and a ribbon element (4.18). This is a quasitriangular Hopf algebra with a ribbon element. It is certainly the proper realization of a quantum group in this example. It was first written down in a paper of Dijkgraaf, Pasquier, and Roche [DPR], and it can be identified with Drinfeld's quantum double of the Hopf algebra of functions on $G$.

Exercise 4.40. Compute the Hopf algebra for $G$ a cyclic group. For $G=S_{3}$.

\section{REFERENCES}

[AC] D. Altschuler, A. Coste, Quasi-quantum groups, knots, three-manifolds, and topological field theory, Commun. Math. Phys. 150 (1992), 83-107.

[DPR] R. Dijkgraaf, V. Pasquier, P. Roche, Quasi-quantum groups related to orbifold models, Nuclear Phys. B. Proc. Suppl. 18B (1990), 60-72.

[DW] R. Dijkgraaf, E. Witten, Topological gauge theories and group cohomology, Commun. Math. Phys. 129 (1990), 393-429.

[F1] D. S. Freed, Higher algebraic structures and quantization, Commun. Math. Phys. 159 (1994), 343-398.

[F2] D. S. Freed, Extended structure in topological quantum field theory, Quantum Topology (Series on Knots and Everything, Volume 3) (L. H. Kauffman, R. A. Baadhio, eds.), World Scientific, Singapore, 1993, pp. 162-173.

[F3] D. S. Freed, Characteristic numbers and generalized path integrals (to appear in proceedings of a conference in honor of Raoul Bott's 70th birthday).

[F4] D. S. Freed, Lectures in topological quantum field theory, Integrable Systems, Quantum Groups, and Quantum Field Theories (L. A. Ibort, M. A. Rodríguez, eds.), Kluwer Academic Publishers, The Netherlands, 1993, pp. $95-156$.

[F5] D. S. Freed, Classical Chern-Simons Theory, Part 1, Adv. Math. (to appear).

[FQ] D. S. Freed, F. Quinn, Chern-Simons theory with finite gauge group, Commun. Math. Phys. 156 (1993), 435-472.

[J] V. F. R. Jones, A polynomial invariant of knots via von Neumann algebras, Bull. Amer. Math. Soc. 12 (1985), 103-112. 
[KV] M. M. Kapranov, V. A. Voevodsky, 2-Categories and Zamolodchikov tetrahedra equations (preprint, 1992).

[L] R. Lawrence, Triangulations, categories and extended topological field theories, Quantum Topology (Series on Knots and Everything, Volume 3) (L. H. Kauffman, R. A. Baadhio, eds.), World Scientific, Singapore, 1993, pp. 191-208.

[Mc] S. MacLane, Categories for the Working Mathematician, Graduate Texts in Mathematics, Volume 5, Springer Verlag, 1971.

[M] Kac-Moody groups and the Dirac determinant line bundle, Topological and geometrical methods in field theory (Espoo, 1986), World Sci. Publishing, Teaneck, NJ, 1986.

[RT1] N. Y. Reshetikhin, V. G. Turaev, Ribbon graphs and their invariants derived from quantum groups, Commun. Math. Phys. 127 (1990), 1-26.

[RT2] N. Y. Reshetikhin, V. G. Turaev, Invariants of 3-manifolds via link polynomials and quantum groups, Invent. Math. 103 (1991), 547-97.

[T] V. G. Turaev, Quantum Invariants of Knots and 3-Manifolds, Walter de Gruyter, Berlin, 1994.

[V] E. Verlinde, Fusion rules and modular transformations in 2d conformal field theory, Nucl. Phys. B300 (1988), 360-376.

[W] E. Witten, Quantum field theory and the Jones polynomial, Commun. Math. Phys. 121 (1989), 351-399.

Department of Mathematics, University of Texas, Austin, TX 78712

E-mail address: dafr@math.utexas.edu 